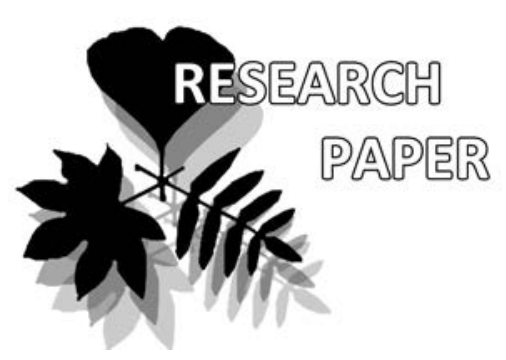

\title{
A contribution to the syntaxonomic diversity of the Tazovsky Peninsula, Arctic Russia
}

\author{
Michael Yu. Telyatnikovi*, Olga V. Khitun², Irina V. Czernyadjeva², \\ Ekaterina Yu. Kuzmina ${ }^{2} \&$ Ksenia A. Ermokhina ${ }^{3}$
}

Michael Yu. Telyatnikov ${ }^{1 *}$ e-mail: arct-alp@mail.ru

Olga V. Khitun ${ }^{2}$

e-mail:khitun-olga@yandex.ru

Irina V. Czernyadjeva ${ }^{2}$

e-mail: irinamosses@yandex.ru

Ekaterina Yu. Kuzmina ${ }^{2}$

e-mail: ekuzmina@yandex.ru

Ksenia A. Ermokhina ${ }^{3}$

e-mail: diankina@gmail.com

${ }^{1}$ Central Siberian Botanical Garden SB RAS, Novosibirsk, Russia

${ }^{2}$ Komarov Botanical Institute RAS,

St. Petersburg, Russia

${ }^{3}$ A.N. Severtsov Institute of Ecology and Evolution RAS, Moscow, Russia

* corresponding author

Manuscript received: 07.12.2020

Review completed: 04.03.2021

Accepted for publication: 20.03.2021

Published online: 24.03.2021

\begin{abstract}
A B S T R A C T
Syntaxonomic diversity of the vegetation in the southern tundra subzone of the Tazovsky Peninsula is represented by six associations, three subassociations and three variants. Four associations (Dicrano maji-Salicetum lanatae Khitun ass. nov. hoc loco, Sphagno girgensohnii-Betuletum nanae Khitun ass. nov. hoc loco, Empetro subholarctici-Eriophoretum vaginati Khitun ass. nov. hoc loco, Eriophoro leiocarpi-Caricetum rotundatae Khitun ass. nov. hoc loco), the three subassociations (Hierochloo alpinaeHylocomietum splendentis empetretosum subholarctici Khitun subass. nov. hoc loco, Eriophoro leiocarpi-Caricetum rotundatae typicum Khitun subass. nov. hoc loco, Eriophoro teiocarpi-Caricetum rotundatae caricetosum chordorrbizae Khitun subass. nov. hoc loco) and the three variants are described for the first time. A high degree of similarity was found between vegetation of the Tazovsky Peninsula and vegetation of the southern part of the Gydansky Peninsula (the typical tundra subzone). They have four of six associations in common, whereas with the northern part of the typical tundra there is only one association in common.
\end{abstract}

Key words : the Arctic, the Tazovsky Peninsula, tundra, mires, vegetation classification, syntaxonomy

\section{P E 3 Ю M E}

Телятников М.Ю., Хитун О.В., Чернядьева О.В., Кузьмина Е.Ю., Ермохина К.А. К синтаксономическому разнообразию Тазовского покуострова, российская Арктика. Синтаксономическое разнообразие южных тунАр северо-восточной части Тазовского полуострова представлено 6 ассоциациями, 3 субассоциациями и 3 вариантами, из которых 4 ассоциации (Dicrano maji-Salicetum lanatae Khitun ass. nov. hoc loco, Sphagno girgensobniiBetuletum nanae Khitun ass. nov. hoc loco, Empetro subholarctici-Eriophoretum vaginati Khitun ass. nov. hoc loco, Eriophoro leiocarpi-Caricetum rotundatae Khitun ass. nov. hoc loco), 3 субассоциации (Hierochloo alpinae-Hylocomietum splendentis empetretosum subholarctici Khitun subass. nov. hoc loco, Eriophoro leiocarpi-Caricetum rotundatae typicum Khitun subass. nov. hoc loco, Eriophoro leiocarpi-Caricetum rotundatae caricetosum chordorrbizae Khitun subass. nov. hoc loco) и 3 варианта описаны впервые. Выявлено высокое сходство растительности района исследований с растительностью южой части типичных тундр Гыланского полуострова (4 из 6 описанных ассоциаций общие) и низкое сходство с растительностью северной части типичных тунАр того же полуострова (общей явАяется всего 1 ассоциация).

КАючевые слова: Арктика, Тазовский полуостров, тунАры, болота, классификация растительности, синтаксономия
There have been a limited number of vegetation studies on the Tazovsky Peninsula. The first such surveys date back to Govorukhin's (1933) inventory of reindeer summer pastures and Nikolaeva's (1941) description of shrub vegetation, both based on dominant approach methodology. An important contribution to the study of the regional vegetation was the creation of the map "Vegetation of the West Siberian Plain" (Il'ina1976) followed by the legend issued as a separate book (Il'ina et al. 1985). However, this book and the map provide only general information, also based on dominant approach. Since 1986, a few studies of zonal differentiation of the plant cover, focused primarily on flora, were carried out on the Tazovsky and Gydansky Peninsulas (e.g., Rebristaya et al. 1989, Khitun 1989, 1998, 2005). The latest research in the area was mainly devoted to the anthropogenic transformation of vegetation, which follows the local gas-oil industry development (e.g., Valeyeva \& Moskovchenko 2008, Moskovchenko et al. 2016, 2017).

Our study is the first application of the floristic-sociological (Braun-Blanquet) approach to the classification of vegetation of the Tazovsky Peninsula. It is also our contribution to the Arctic Vegetation Archive international project (Walker et al. 2018; https://avarus.space).

The aim of our study is to classify southern tundra subzone vegetation and identify syntaxonomic diversity in the northwest of the Tazovsky Peninsula.

\section{Study area}

Geology and topography. The Tazovsky Peninsula, as well as the Gydansky Peninsula, lies in the north of the West Siberian Plain. The terrain is formed by the 200 to $250 \mathrm{~m}$ thick layer of fine-grained Quaternary sedimentary depo- 
sits. The geomorphology reflects the Pleistocene - Holocene marine transgressions and regressions. Overall, five geomorphological levels (terraces) are distinguished in the West Siberian Arctic. At the study site, the 3rd marine terrace with an elevation 30 to $45 \mathrm{~m}$ above sea level (a.s.l.) is present (Ogorodnov et al. 1971). The topography is represented by flat gently rolling watershed hills dissected by water tracks and meandering streams. Lakes and wetlands are numerous both in riverine valleys and on poorly drained areas of lower watershed hills. The region lies entirely within the zone of continuous permafrost and therefore the area shows many cryogenic features, such as nonsorted circles and earth hummocks, ice-wedge polygons, high-centered polygonal tundra-mire complexes, thermokarst lakes and drained thermokarst basins, locally called "khasyrei". Deep ravines form at the edges of the terrace starting from the ice wedge thaw. Sandy and loamy deposits predominate in the study area. On the watersheds formed by sandy deposits, areas of eroded sand lacking vegetation (sometimes rather extended) appear due to deflation. A consequence of waterlogging of soils is accumulation of the thick peat horizons, which is the most general characteristic of subarctic tundra in West Siberia (Khitun 2005).

Climate. The area belongs to the arctic belt in the Region of the Atlantic influence (Antonov et al. 1986). Cyclonic circulation causes large diurnal amplitudes of air temperature, high frequency of strong winds and cloudy sky, as well as relatively intense precipitation (Simonov 1977). According to: https://weatherarchive.ru/Pogoda/ Yamburg (28.06.2020) mean annual air temperature in Yamburg (the closest station to the study site, see Fig. 1) for the period from 2014 to 2019 is $-5.9^{\circ} \mathrm{C}$. The mean July temperature is $+13.1^{\circ} \mathrm{C}$; mean January temperature is $-22.9^{\circ} \mathrm{C}$. Precipitation in this region is $330-380 \mathrm{~mm}$, of which $75 \%$ falls during summer and autumn (Ogorodnov et al. 1971).

Zonal position and general overview of vegetation. The study area is located within the southern tundra subzone (Gorodkov 1935, Chernov \& Matveyeva 1997), which is also called the southern hypoarctic tundra subzone (Yurtsev 1994) and it coincides with subzone E, or the lowshrub subzone on the Circumpolar Arctic Vegetation Map (CAVM Team 2003). In the system of geobotanical division of the Arctic by Aleksandrova (1980), the subarctic tundra is divided into three belts (in contrast to the mentioned above schemes with only two subdivisions) and the study area belongs to the southern belt of the subarctic tundra subzone, at its border with the middle belt. We compare our associations with those distinguished previously on the Gydansky Peninsula (Telyatnikov et al. 2019) in the typical tundra subzone (Gorodkov 1935, Chernov \& Matveyeva 1997). The latter is also called the northern hypoarctic tundra (Yurtsev 1994) and it coincides with subzone D, or the erect dwarf shrub subzone (CAVM Team 2003). According to the system of Aleksandrova (1980) that study was carried out in the northern belt of the subarctic tundra subzone. Hereafter we use the abbreviations: STS, southern tundra subzone; TTS, typical tundra subzone.

The characteristic feature of vegetation in the STS is the predominance of low deciduous shrubs, primarily Betu-

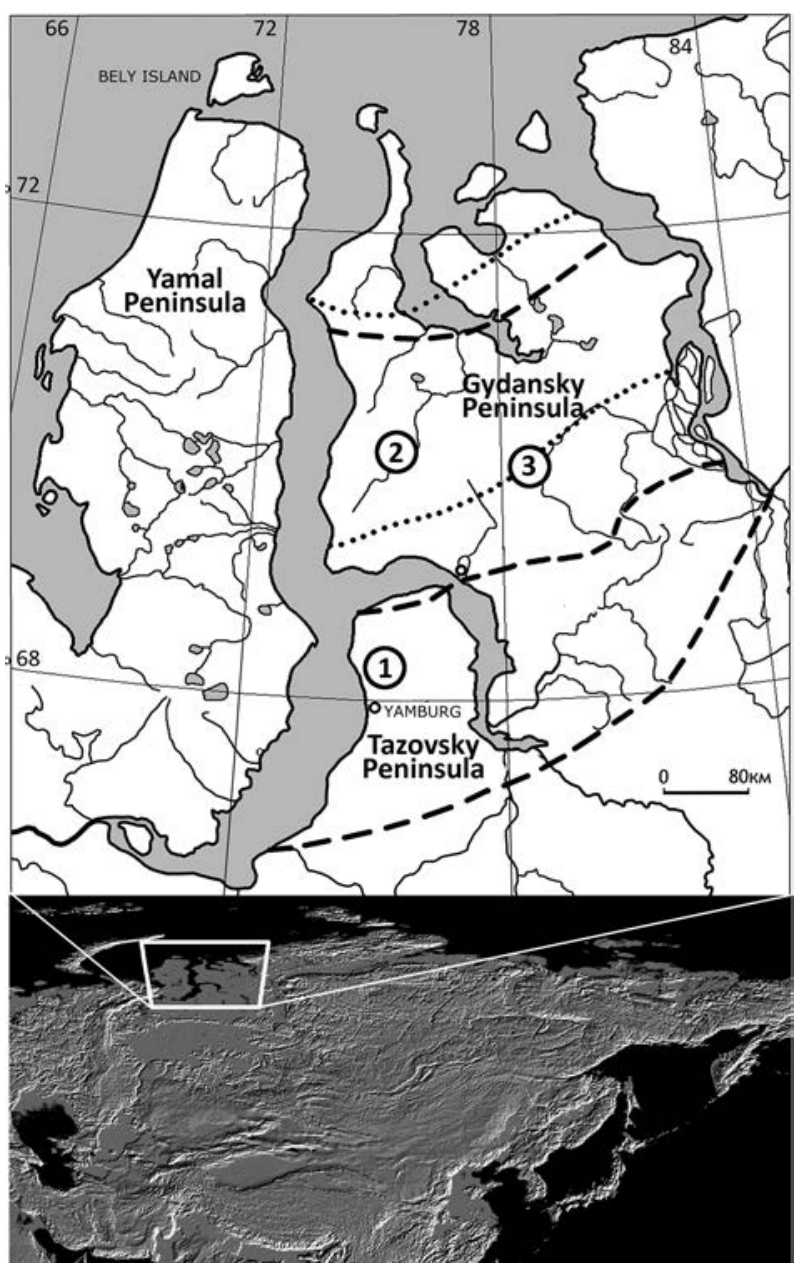

Figure 1 Study sites (circled numbers): 1 - upper reaches of the Verkhnyava Yareiyakha River; 2 - Lake Parisento surroundings; 3 basin of the Tanama River middle reaches. Our field-work revealed that the boundaries of the TTS should be essentialy shifted northwards compare to their position in Yurtsev (1994): dashed lines - borders as in Yurtsev (1994); dotted lines - borders according to Khitun (2005), Telyatnikov et al. (2019) and Telyatnikov et al. (in print)

la nana and to a lesser extent Salix glauca and S. lanata, in zonal communities on watershed hills. Erect dwarf shrubs Vaccinium uliginosum subsp. microphyllum, V. vitis-idaea subsp. minus, Ledum palustre subsp. decumbens are present and abundant in the majority of communities except those that are permanently waterlogged. Tall (up to $2.5 \mathrm{~m}$ ) willow thickets (Salix lanata, S. glauca, S. phylicifolia) with herbaceous understory occupy the relatively steep slopes of hollows and lake basins and riparian habitats. Lower (1-1.5 m) willow thickets with developed herb-moss understory usually grow at the bottom of wider hollows. Alder (Alnus fruticosa) in the studied locality was found rarely, as solitary shrubs on slopes. Oligotrophic hypoarctic and boreal species dominate in all types of vegetation in this subzone. Proper arctic species grow either in snowbeds (Ranunculus nivalis, Salix polaris, S. arctica) or on the edges of exposed, windblown hilltops (Armeria maritima, Hierochloë alpina, Salix nummularia, Pachypleurum alpinum).

Various variants of low- and dwarf-shrub dominated tundra with a well-developed lichen-moss ground layer are widespread on watershed hills with a better drainage. 
Dwarf birch-Sphagnum spp. communities with very dense shrub layers populate the slope edges of low watershed hills and lake terraces. Betula nana + Ledum palustre s. 1. + Eriophorum vaginatum communities with thick moss mats cover large flat-centered polygons (30 to $40 \mathrm{~cm}$ high) in the tundra-mire complexes, which are common on the lower watersheds with a poorer drainage. Sedge (Carex rotundata, Eriophorum angustifolium) - moss mires occur in the troughs between the polygons. Sedge-moss mires dominated by Carex rotundata and C. chordorrbiza, cover the bottoms of drained thermokarst basins, whereas Carex concolor dominated communities with or without a moss layer occupy more saturated habitats, along water tracks and streams. Psammophytic communities with abundant grasses, herbs and dwarf shrubs grow on various eroded steep sandy slopes.

\section{MATERIAL AND METHODS}

\section{Sampling and data analysis}

The study site is located approximately $35 \mathrm{~km}$ to the north of Yamburg settlement, in the upper reaches of the Verkhnyaya Yareiyakha River at $68^{\circ} 13^{\prime} \mathrm{N} 75^{\circ} 12^{\prime} \mathrm{E}$ (Fig. 1, site 1). Olga Khitun conducted vegetation sampling between 27 July and 8 August 2017. Plots locations were subjectively chosen in areas of homogenous vegetation in visually different communities along several transects established in the area. Plot sizes varied from 25 to $100 \mathrm{~m}^{2}$. In total, 65 relevés were sampled, 37 of which are used in this paper. Between 8 July and 7 August 2017, Olga Khitun carried out similar investigations at two sites in the southern part of the TTS on the Gydansky Peninsula: in the surroundings of the Parisento Lake, $70^{\circ} 06^{\prime} \mathrm{N} 75^{\circ} 36^{\prime} \mathrm{E}$ (Fig. 1, site 2) and in the basin of the Tanama River middle reaches, $69^{\circ} 56^{\prime} \mathrm{N} 78^{\circ} 50^{\prime} \mathrm{E}$ (Fig. 1, site 3). At those sites, 215 relevés were sampled, 75 of them are used in another publication (Telyatnikov et al. in press). However, initially all relevés were analysed together in MegaTab (see below), and 19 relevés from the Gydansky Peninsula are considered here because they belong to synataxa common for both subzones.

We use the modified Braun-Blanquet cover-abundance scale (Mirkin \& Naumova 1998) to score the cover of each species: $1<1 \% ; 2=1-5 \% ; 3=6-10 \% ; 4=11-25 \% ; 5=$ $26-50 \% ; 6=51-75 \% ; 7=76-100 \%$. At each relevé, we recorded the complete species composition (including mosses and lichens) and cover-abundance scores for each species. The percentage cover of major plant growth forms was visually estimated. Canopy height, thickness of moss layer and depth of soil organic horizons were measured with a ruler. Soil texture was estimated according to Bogolubov et al. (2001) after digging small soil pits. Coordinates and altitude of plots were taken with Garmin e'Trex 10.

We classified the vegetation according to the BraunBlanquet sorted-table method (Westhoff \& van der Maarel 1978). The relevés were entered in the TURBOVEG database (Hennekens \& Schaminée 2001), thereafter a sorted table was derived in the MegaTab program (Hennekens 1996) and a dichotomous hierarchy of groups of relevés was constructed using the TWINSPAN program (Hill 1979) and transformed by M.Yu. Telyatnikov into a hierarchy of syntaxa. At this stage, we identified diagnostic (character), differential and constant species; we discarded transitional, between syntaxa, relevés.

\section{Nomenclature}

The nomenclature of the described syntaxa is in accordance with the International Code of Phytosociological Nomenclature (Theurillat et al. 2021). Diagnostic species of the class Loiseleurio procumbentis-Vaccinietea Eggler ex Schubert 1960, are given in accordance with "Vegetation of Europe..." (Mucina et al. 2016) and the paper of Ermakov (2012). Diagnostic species of the class OxycoccoSphagnetea Br.-Bl. et Tx. ex Westhoff et al. 1946 are cited after Lavrinenko \& Lavrinenko (2015) and also "Vegetation of Europe..." (Mucina et al. 2016) and of the class Scheuchzerio-Caricetea nigrae (Nordh. 1936) Tx. 1937 - after Lavrinenko et al. (2016).

The nomenclature of the species followed Sekretareva (2004) for vascular plants, Ignatov et al. (2006) for mosses, Potemkin \& Sofronova (2009) for liverworts and Esslinger (2016) for lichens.

The synoptic table presents syntaxa with their constancy class in each community: $+=$ present in 1 to $10 \%$ of records; I $=11-20 \%$; II $=21-40 \%$; III $=41-60 \%$; IV = 61-80\%; V = 81-100\%.

In the description of nomenclatural type relevés (holotypes), to avoid the repetition of headings and long names of localities throughout this paper we write in the following order: 1) relevé number in the table and, in parentheses, in the database, for example, 3(276);

2) table No (T1 for Table 1 or T2 for Table 2);

3) region is Yamal-Nenets Autonomous Area (YaNAA);

4) locality is indicated by number as in Figure 1 (1, VerkhnyayaYareiyakha; 2 , Parisento; 3 , Tanama);

5) coordinates (latitude, $\mathrm{N}$ and longitude, E);

6) elevation, $\mathrm{m}$ above sea level (a.s.l.);

7) plot surface area $\left(\mathrm{m}^{2}\right.$, most commonly it was $\left.100 \mathrm{~m}^{2}\right)$.

This is followed by the information about habitat (topography, soil, cover of growth forms); date of observation (ddmmyyyy); author; and, finally, list of species in the typus relevé with cover abundance scores.

\section{RESULTS}

\section{Dwarf shrub tundra in relatively dry wind-exposed habitats}

We placed tundra communities of relatively dry habitats in the class Loiseleurio procumbentis-Vaccinietea Eggler ex Schubert 1960, order Deschampsio flexuosae-Vaccinietalia myrtilli Dahl 1957 and alliance Loiseleurio-Arctostaphylion Kalliola ex Nordhagen 1943.

The Loiseleurio procumbentis-Vaccinietea includes dwarf shrub vegetation on dry acidic habitats with thin snow cover in alpine and arctic regions of the northern hemisphere. Diagnostic species of the class are: Alectoria ochroleuca, Aulacomnium turgidum, Betula nana, Cladonia bellidifolia, C. stellaris, Diphasiastrum alpinum, Flavocetraria cucullata, F. nivalis, Hierocbloë alpina, Huperzia arctica, Lycopodium annotinum subsp. pungens, L. clavatum subsp. monostachyon, Ochrolechia frigida, Pedicularis 
lapponica, Stereocaulon paschale, Sphaerophorus globosus, Vaccinium vitis-idaea subsp. minus, $V$. uliginosum subsp. microphyllum.

The Deschampsio flexuosae-Vaccinietalia myrtilli includes dwarf shrub or low shrub dominated tundra vegetation of the Arctic and mountain tundra of the northern Eurasia and the islands and archipelagos of the Arctic Ocean. Diagnostic species of the order are: Betula nana, Carex arctisibirica.

The Loiseleurio-Arctostaphylion includes mountain and lowland tundra of northern Eurasia, Svalbard, Island and Greenland. Diagnostic species of the alliance are Alectoria ochroleuca and Flavocetraria nivalis. Within this alliance, we place two variants and one subassociation of the association described earlier.

Hierochloo alpinae-Hylocomietum splendentis (Table 1, rel. 1-14; Table 3)

Diagnostic species: Hierochlö̈ alpina, Polytrichum byperboreum, Alectoria ochroleuca;

Differential species: Festuca ovina, Luzula confusa, Salix nummularia, Bryocaulon divergens, Flavocetraria nivalis, Bryoria nitidula, Racomitrium lanuginosum.

Distribution and ecology. This association is described from the northern part of the TTS on the Gydansky Peninsula (Telyatnikov et al. 2019). These communities occupy dry, often slightly convex, marginal parts of the hilltops and adjacent upper parts of slopes with shallow snow accumulation.

Structure and composition. Such communities in Russian literature are commonly called "spotted" tundra because they have complex horizontal structure including small patches of bare ground surrounded by vegetated rims and small troughs. Communities have a sparse upper layer (up to $20 \mathrm{~cm}$ high) of grass straw and closed ground layer formed by prostrate dwarf shrubs and cryptogams. The cover values vary between 25 and $55 \%$ of dwarf shrubs, 20 to $60 \%$ of lichens and 30 to $70 \%$ of mosses (Telyatnikov et al. 2019).

Hierochloo alpinae-Hylocomietum splendentis empetretosum subholarctici subass. nov. hoc loco (Table 1, rel 1-14; Table. 3, Fig. 2A)

Differential species: Empetrum subholarcticum, Arctous alpina, Calamagrostis neglecta.

Holotypus: 3 (276); T1; YaNAA; 1; 68¹2'25.8"N 75²1'25.5"E; $47 ; 100$.

Relevé. Well-drained marginal part of the hilltop with expressed polygonal cracks; dry nonsorted circles and small earth hummocks are almost completely overgrown with vegetation. Inclination $2^{\circ}$. Aspect $202^{\circ}$. Soil: the dark brown peaty humus organic horizon is $1-2 \mathrm{~cm}$ thick and the mineral horizon is light yellowish-brown with a sandy texture. The cover of lichens is $70 \%$, of mosses $30 \%$, shrubs $15 \%$, dwarf-shrubs $10 \%$, herbs $15 \%$. 04.08.2017. O.V. Khitun.

List of species: Alectoria ochroleuca 5, Polytrichum byperboreum 4, Bryocaulon divergens 4, Betula nana 4, Cladonia stygia 3, Calamagrostis neglecta 3, Carex arctisibirica 3, Dicranum elongatum 3,
Polytrichum juniperinum 3, Alectoria nigricans 2, Bryoria nitidula 2, Cladonia gracilis s. 1. 2, Cetraria islandica 2, Flavocetraria cucullata 2, Hierocbloë alpina 2, Ochrolechia frigida 2, Pogonatum dentatum 2, Salix glauca 2, Vaccinium uliginosum subsp. microphyllum 2, Arctous alpina 1, Aulacomnium turgidum 1, Bistorta elliptica 1, B. vivipara 1, Cladonia arbuscula 1, C. chlorophaea 1 , C. cornuta 1, C. pleurota 1, Cetraria laevigata 1, C. aculeata 1, Eriophorum vaginatum 1, Empetrum subholarcticum 2, Festuca ovina 2, Flavocetraria nivalis 1, Gymnomitrion coralloides 1, Sphenolobus minutus 1, Ledum palustre subsp. decumbens 2, Luzula confusa 1, Pedicularis labradorica 1, Pertusaria panyrga 1, Pleurozium schreberi 1, Poa alpigena 1, Poblia sp. 1, Salix nummularia 1, S. pulchra 1, Vaccinium vitis-idaea subsp. minus 1.

Distribution and ecology. The communities of the subass. empetretosum subholarctici occur in the STS on the Tazovsky Peninsula and in the southern part of the TTS on the Gydansky peninsula. They occupy well-drained parts of watersheds such as the edges of hilltops, the upper parts of hill slopes and river terraces; at altitudes varying between 24 and $57 \mathrm{~m}$ a.s.l. Compared to the association, in subassociation the cover of shrubs and lichens is higher, and the cover of herbs, dwarf shrubs and mosses is lower.

The subassociation splits into two variants.

Variant typicum (Table 1, rel. 1-6; Table 3; Fig. 2A)

Differential species are the same as for the subassociation.

Distribution and ecology. These communities occur in the STS on the Tazovsky Peninsula on well-drained parts of watersheds and upper parts of their slopes with an inclination of 1 to $15^{\circ}$ and within an altitude range of 43 to $57 \mathrm{~m}$. The microtopography is polygonal with flat polygons 15 to $20 \mathrm{~m}$ in diameter divided by cracks of approximately $0.5 \mathrm{~m}$ in width. The organic horizons range in thickness from 2 to $3 \mathrm{~cm}$ and consist of dark brown mucky peat; the underlying mineral ground is yellow sand or sandy loam.

Structure and composition. These communities have two vertical strata. Low shrubs, predominantly dwarf birch, (15 to $25 \%$, rarely $5 \%$ ) and herbs, predominantly Calamagrostis neglecta and Carex arctisibirica, (10 to $25 \%$ ) form a sparse evenly distributed overstory, usually 10 to $15 \mathrm{~cm}$ height. The dwarf shrub-cryptogam understory is closed, with lichens cover of 70 to $90 \%$ that serves as a background for mosses (up to $35 \%$ ) and separate patches (5 to $10 \%$ ) of prostrately growing in this conditions dwarf shrubs such as Ledum palustre subsp. decumbens, Vaccinium uliginosum subsp. microphyllum, V. vitis-idaea subsp. minus. Alectoria ochroleuca and Cladonia stygia are the most abundant among the lichens, and Polytrichum hyperboreum and Dicranum elongatum are predominant among the mosses.

Variant Aconogonon ochreatum (Table 1, rel. 7-14; Tabl. 3; Fig. 2B)

Differential species: Tanacetum bipinnatum, Aconogonon ochreatum, Carex quasivaginata, Campanula rotundifolia.

Distribution and ecology. These phytocoenoses occur in the southern part of the TTS on the Gydansky Peninsula and in the STS on the Tazovsky Peninsula. They occupy windswept marginal parts of watershed hills and the upper

Figure 2 Photographs of the described plant communities on the Tazovsky Peninsula. A - Hierochloo alpinae-Hylocomietum splendentis subass. empetretosum subbolarctici var. typicum on well-drained part of the watershed hill; B - Hierocbloo alpinae-Hylocomietum splendentis subass. empetretosum subholarctici var. Aconogonon ocbreatum on the gentle convex slope of the watershed hill; C - Dicrano maji-Salicetum lanatae on the bottom of the hollow at the watershed hillslope; D - Sphagno girgensohnii-Betuletum nanae on the lake terrace; E - Empetro subholarctici-Eriophoretum vaginati on the polygons in tundra-mire complex on the flat low watershed; F-Eriophoro leiocarpi-Caricetum rotundatae subass. typicum in the wet trough between polygons of tundra-mire complex; G - Eriophoro leiocarpi-Caricetum rotundatae subass. caricetosum chordorrbizae on the bottom of the drained lakebed; H - Carici stantis-Warnstorfietum exannulatae var. Warnstorfia sarmentosa on the bottom of the wet hollow in the watershed hill slope 

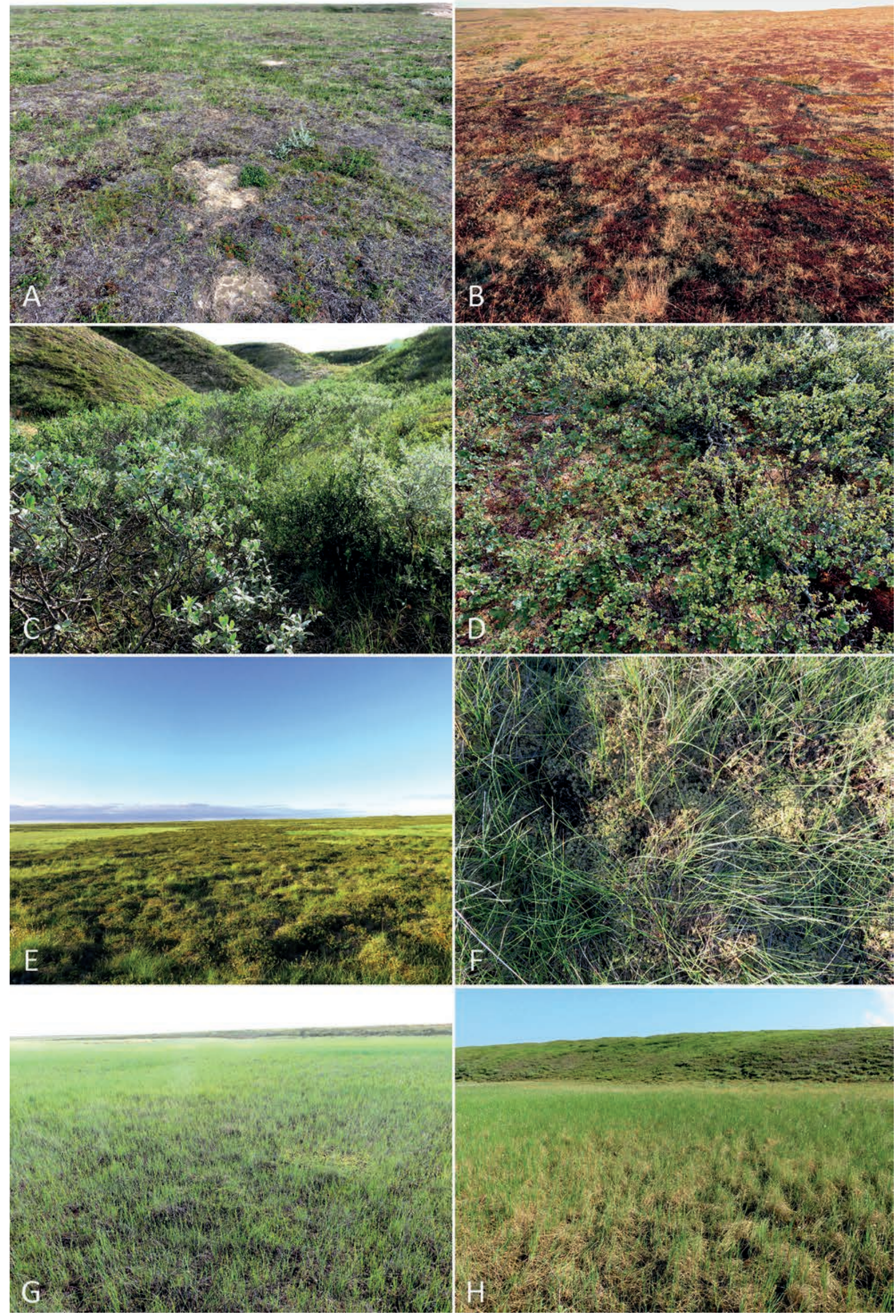
Telyatnikov et al.

Table 1. Associations Hierochloo alpinae-Hylocomietum splendentis (var. typicum - A, and var. Aconogonon ochreatum - B), Dicrano majiSalicetum lanatae $(\mathbf{C})$, Sphagno girgensohnii-Betuletum nanae $(\mathbf{D})$ and Empetro subholarctici-Eriophoretum vaginati $(\mathbf{E})$

\begin{tabular}{|c|c|c|c|c|c|c|c|c|c|c|}
\hline SYNTAXON CODE & A & B & $\mathbf{C}$ & $\mathbf{D}$ & $\mathrm{E}$ & A & B & $\mathbf{C}$ & $\mathbf{D}$ & E \\
\hline Locality & \multirow{8}{*}{\multicolumn{5}{|c|}{ 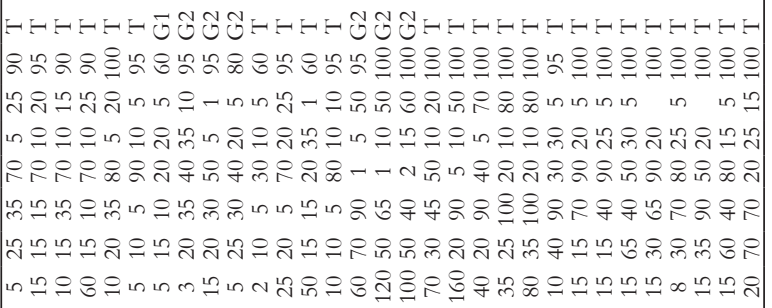 }} & & & & & \\
\hline Cover total, $\%$ : & & & & & & & & & & \\
\hline Cover shrubs, $\%$ & & & & & & & & & & \\
\hline Cover dwarf shrubs, $\%$ & & & & & & & & & & \\
\hline Cover lichens, $\%$ & & & & & & & & & & \\
\hline Cover mosses, $\%$ & & & & & & & & & & \\
\hline Cover herbs, $\%$ & & & & & & & & & & \\
\hline Height shrubs (cm) & & & & & & & & & & \\
\hline Aspect $\left({ }^{\circ}\right)$ & \multicolumn{5}{|c|}{ 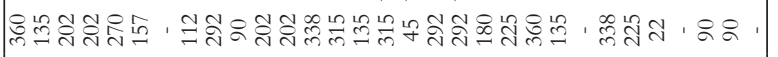 } & & & & & \\
\hline Inclination of slope $\left(^{\circ}\right)$ & \multicolumn{5}{|c|}{ 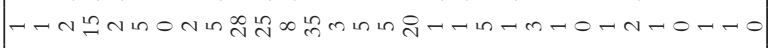 } & & & & & \\
\hline Altitude ( $\mathrm{m}$ a.s.l. $)^{1}$ & \multirow{7}{*}{\multicolumn{5}{|c|}{ 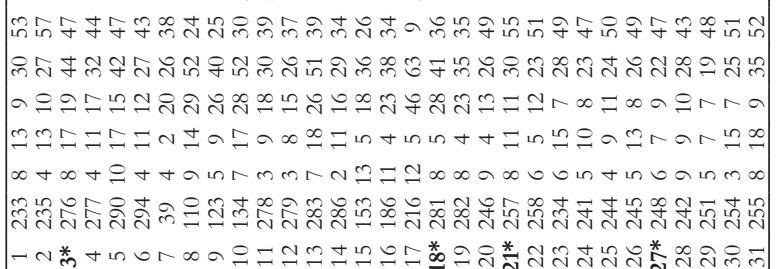 }} & & & & & \\
\hline Total number of species & & & & & & & & & & \\
\hline Number of vascular plants species & & & & & & & & & & \\
\hline Number of lichen species & & & & & & & & & & \\
\hline Number of moss species & & & & & & & & & & \\
\hline Relevé nr: in the database & & & & & & & & & & \\
\hline $\begin{array}{l}\text { In the table } \\
\text { Number of relevés }\end{array}$ & & & & & & 6 & 8 & 6 & & \\
\hline
\end{tabular}

Diagnostic species of Hierochloo alpinae-Hylocomietum splendentis Hierochloë alpina (L-V) Alectoria ochroleuca (L-A, L-V)

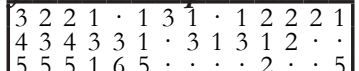

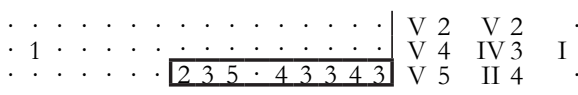

Differential species of Hierochloo alpinae-Hylocomietum splendentis Festuca ovina Luqula confusa Salix nummularia

Bryocaulon divergens Flavocetraria nivalis (L-A, L-V, Rc-De)
Bryoria nitidula

Racomitrium lanuginosum (Sh)

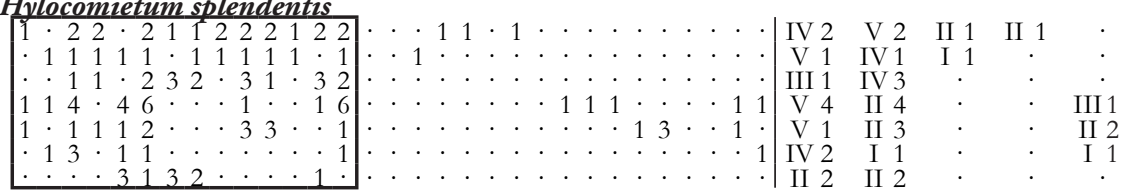

Differential species in subass. empetretosum subbolarctici and var. typicum Empetrum subbolarcticum $\quad \begin{array}{lllllllllllll}2 & 2 & 2 & 2 & 1 & 2 & 1 & 3 & 2 & 2 & 2 & 2 & 4\end{array}$ Arctous alpina Calamagrostis neglecta

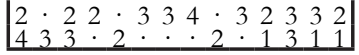

Differential species in var. Aconogonon ochreatum

Tanacetum bipinnatum Aconogonon ochreatum Carex quasivaginata Campanula rotundifolia
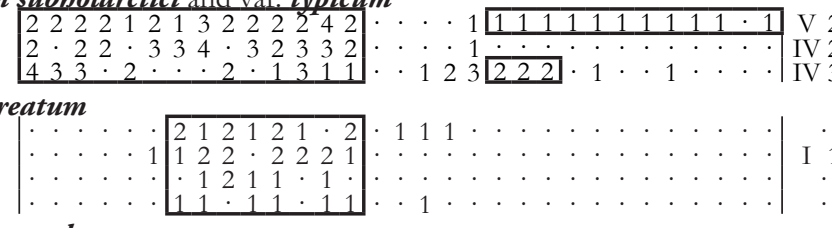

$\begin{array}{llllllll}2 & \mathrm{~V} & 3 & \mathrm{I} & 1 & \mathrm{~V} & 1 & \mathrm{~V} 1 \\ \mathrm{~V} & 2 & \mathrm{~V} & 3 & \mathrm{I} & 1 & & .\end{array}$

ifferential species in Dicrano maji-Salicetum lanatae Carex stans

Dicranum majus

Polemonium acutiflorum

Aulacomnium palustre (O-S)

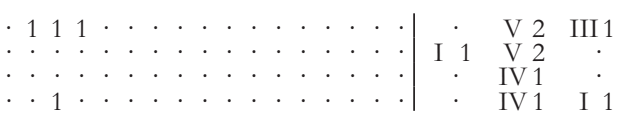

Aulacomnium palustre (O-S)

Sphagnum girgensobnii
Polytrichum commune

Differential species in.Empetro subholarctici-Eriophoretum vaginati Cladonia stellaris (L-V)

Sphagnum lenense

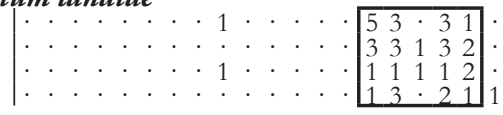

I 1 IV 4 II 1

I 1 V 11

IV 2 II 1

Diagnostic combination of species in alliance Rubo chamaemori-Dicranion elongati (Rc-De)

Cetraria islandica

Dicranum elongatum

Ledum palustre subsp. decumbens

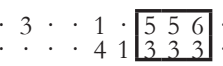

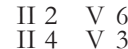

I 3

Diagnostic species in class Salicetea herbaceae (Sh)

Salix polaris

Carex lachenalii

Conostomum tetragonum

Poblia drummondir

Ranunculus nivalis

Ranunculus pyomaens

Sibbaldia procumbens

14.41 . 4 . . . . . . .

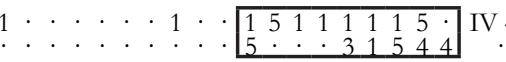

II 4

II 1 V 3

Diagnostic species in order Deschampsio flexuosae-Vaccinietalia myrtilli (D-V)

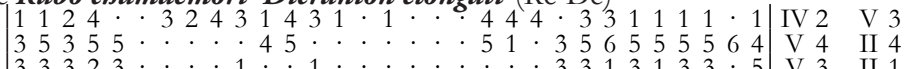

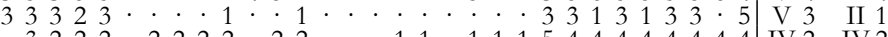

$\mathrm{V} 4$ IV 2

IV 4 V 5

II 1 V 1 V 4

Diagnostic species in class Loiseleurio procumbentis-Vaccinietea (L-V)

Betula nana (D-V, O-S, Rc-De)

Vetula nana (D-V, O-S, Rc-De)
Flavocetraria cucullata

accinium vitis-idaea subsp. minus (Rc-De)

Aulacomnium turgidum

Lycopodium annotinum subsp. pungens

Ochrolechia frigida

Sphaerophorus globosus

Cladonia bellidiflora

Diphasiastrum alpinum

Lycopodium clavatum subsp. monostachyon

Pedicularis lapponica

Stereocaulon paschale

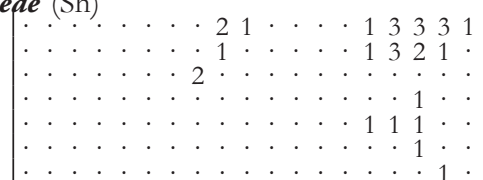

II 2 V 3

I 12 IV 2

$\begin{array}{rrr}\text { I } & 1 \\ \text { III } & 1 \\ \text { I } & 1\end{array}$

I 1 
Table 1 Continued.

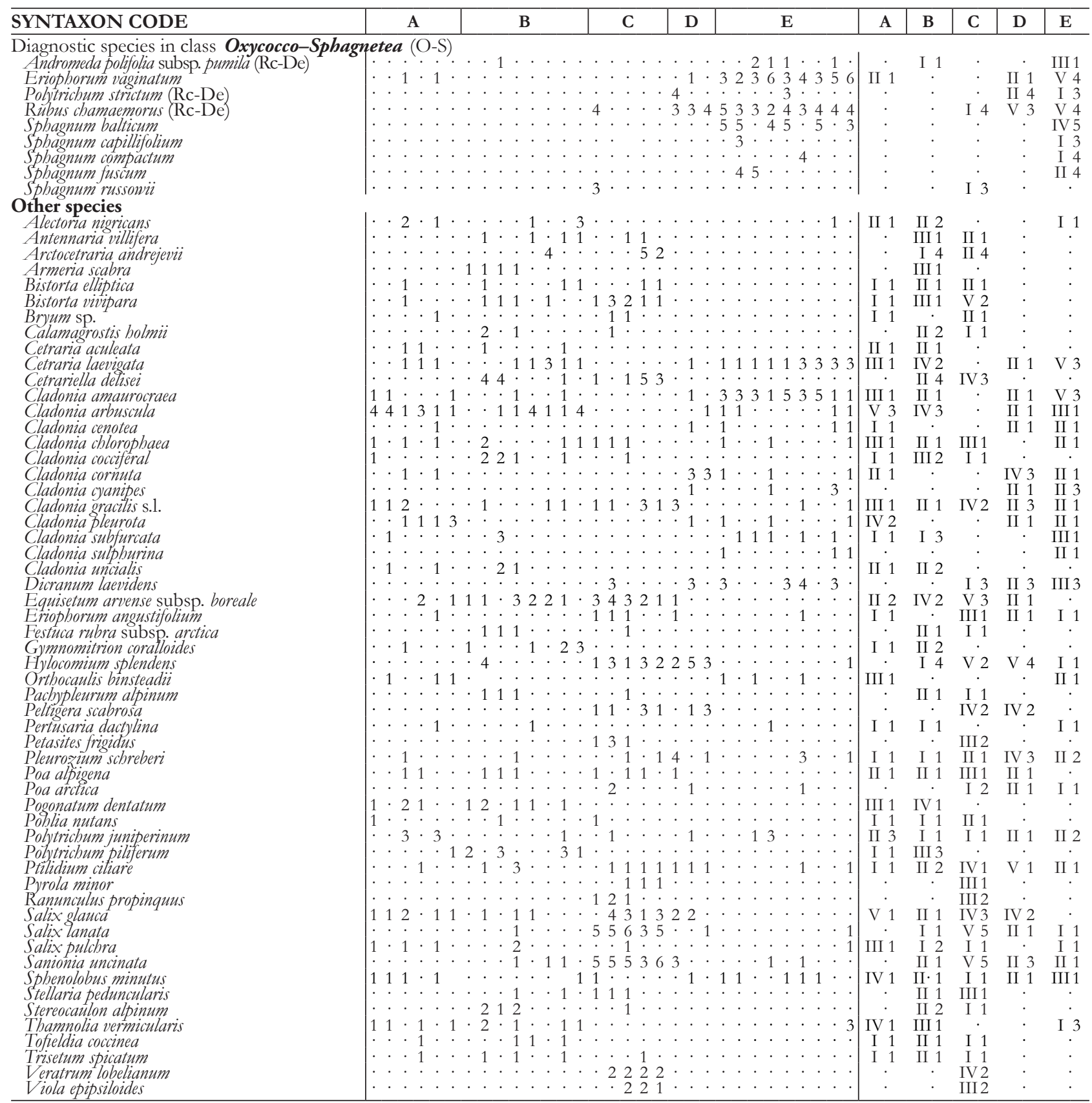

Note. Single occurrence: Alopecurus alpinus 17(1); Arctagrostis latifolia 5(1); Artemisia borealis 7(1); A. tilesii 16(1), 17(1); Astragalus alpinus subsp. arcticus 17(1); Calamagrostis lapponica 4(1), 22(1); C. neglecta subsp. groenlandica 4(2), 6(1); Carex aquatilis 22(3); C. rariflora 18(1), 30(1); C. rotundata 5(1), 29(1); Cerastium jenisejense $17(1)$; Corallorrbiza trifida 17(1); Dryas octopetala subsp. subincisa $7(2)$, 8(2); D upontia fischeri 15 (1); Eremogone polari $13(1)$; Eriophorum russeolum subsp. leiocarpum 25(1); E. russeolum subsp. russeolum 15(2); E. scheuch zeri 16 (1); Hedysarum hedysaroides subsp. arcticum $17(1)$; Juncus biglumis $9(1)$; J. trifidus 13(1); Lagotis minor 8(1), 17(1); Luzula multiflora subsp. frigida 18(1), 19(1); L. wablenbergii 15(1); Lycbnis sibirica subsp. samojedorum 7(1); Myosotis asiatica 17(1); M. palustris 19(1); Oxyria digyna 10 (1), 17(1); Parnassia palustris subsp. neogaea 17 (1); Pediculari compacta 17(1); P. hirsuta 8(1); P. interioroides 16(1), 17(1); P. labradorica 3(1), 18(1); Pinguicula vulgaris 25(1); Polemonium boreale $7(1)$, $14(1) ;$ Pyrola rotundifolia $9(1) ; \dot{R}$ umex acetosa subsp. pseudoxyria $13(1) ; R$. graminifolius $7(1) ;$ Salix arctica $16(1) ; S$. lapponum $19(1) ; S$. myrtilloides $5(1) ; S$. phylicifolia 19(2), 22(1); S. reptans 17(1), 26(1); Saxifraga cernua 17(1); S. bieracifolia 16(1); S. nelsoniana 17.(1); Solidago lapponica 13(1), 18(1); Trisetum sibiricum subsp. litorale 17(1); Trollius asiaticus 17(1); Valeriana capitata 8(1). Lichens: Arthrorbaphis alpina 10(1); Asabinea chrvsantha 10(1); Cetraria nigricans 6(1),13(1); Cladonia deformis 23(1); C. macrophylla 30 (1); C. verticillata-vulcani 6(1), 13(1); Dactylina arctica $5(1)$, 10(1); Icmadophila ericetorum 23(1); Lobaria linita 8(1); Peltigera aphthosa 22(1), 31(1); P. leucophlebia 8(1), 17(1); P. malacea 13(1), 22(1); P. rufescens 8(1); Pertusaria panyrga 3(1);

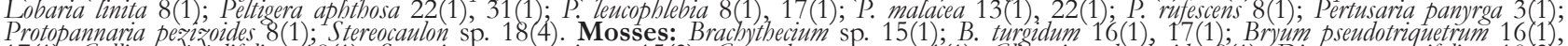

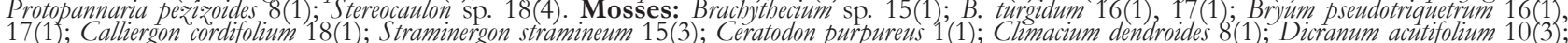
D. spadiceum 8(3), 9(1); Distichium capillaceum 1(1); Kiaeria glacialis $9(4) ;$ Oncophorus wablenbergii $5(1), 15(1) ;$ Paludella squarrosa $15(3) ;$ Plagiomnium cuspidatum 16(1), 17(1); Pohlia sp. 3(1), 10(1); Polytrichastrum alpinum 30(1); Polytrichum jensenii 28(3), 29(1); P. longisetum 5(1).

Author: O.V. Khitun. Localities: Yamal-Nenets Autonomous Area. T - the Tazovsky Peninsula, upper reaches of the Verkhnyaya Yareiva-

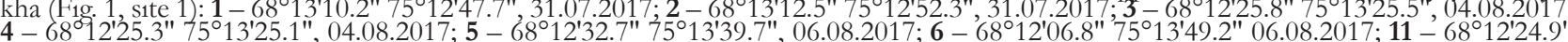

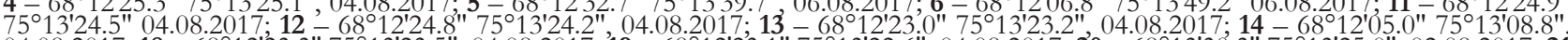

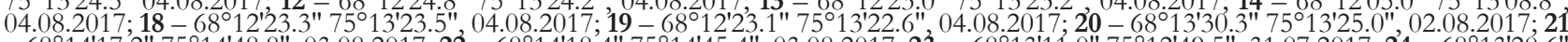

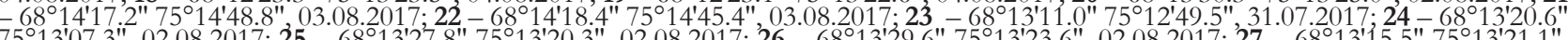

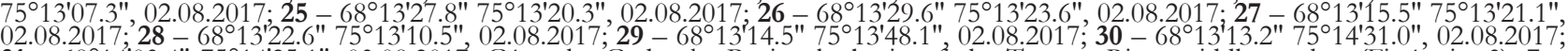
31 - 68 14'03.4" 75 14'25.1", 03.08.2017; G1 - the Gydansky Peninsula, basin of the Tanama River middle reaches (Fig. ' $^{\circ}$ site 3): 7 -

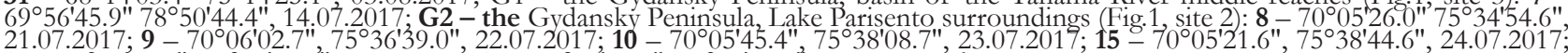

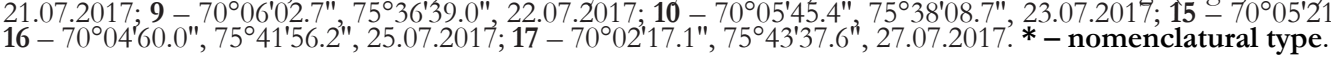


Table 2. Associations Eriophoro leiocarpi-Caricetum rotundatae (subass. typicum - F, subass. caricetosum chordorrbizae - $\mathbf{G}$ ) and Carici stantis-Warnstorfietum exannulatae $(\mathbf{H})$

\begin{tabular}{|c|c|c|c|c|}
\hline SYNTAXON CODE & $\mathbf{H}$ & $\mathbf{F}$ & G & $\mathbf{H}$ \\
\hline Locality & 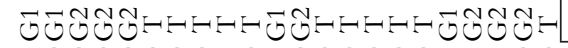 & & & \\
\hline Cover total, $\%$ : & 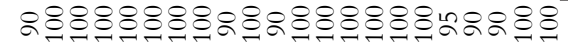 & & & \\
\hline Cover shrubs, $\%$ & 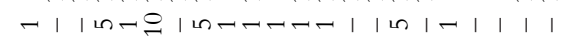 & & & \\
\hline Cover dwarf shrubs, $\%$ & 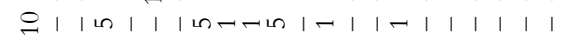 & & & \\
\hline Lichens, $\%$ & $\begin{array}{llllllllllllllllllllll}1 & 1 & 1 & - & 1 & 0 & 1 & 1 & 1 & 1 & 1 & 1 & 1 & 1 & 1 & 1 & 1 & 1 & 1 & 1 & 1 & 1\end{array}$ & & & \\
\hline Mosses, $\%$ & 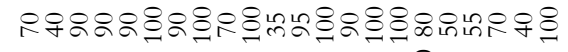 & & & \\
\hline Herbs, $\%$ & 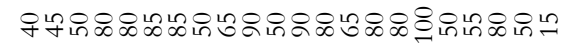 & & & \\
\hline Height shrubs $(\mathrm{cm})$ & 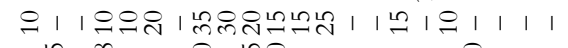 & & & \\
\hline Aspect $\left({ }^{\circ}\right)$ & 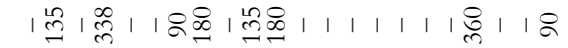 & & & \\
\hline Inclination of slope $\left(^{\circ}\right)$ & $0-0-00-n 0 n-0000000-00-$ & & & \\
\hline Altitude (m a.s.l.) & 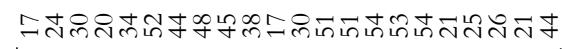 & & & \\
\hline Total number of species & 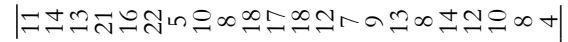 & & & \\
\hline Number of vascular plant species & 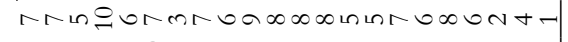 & & & \\
\hline Number of lichens species & 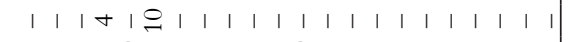 & & & \\
\hline Number of moss species & 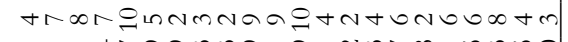 & & & \\
\hline Relevé nr.: in the database & 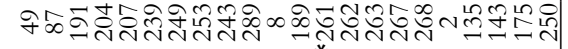 & & & \\
\hline Relevé nr.: in the table & 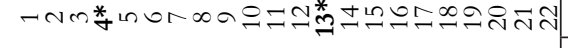 & & & \\
\hline Number of relevés & & 9 & 8 & 5 \\
\hline
\end{tabular}

Differential species in Eriophoro leiocarpi-Caricetum rotundatae and subass. typicum

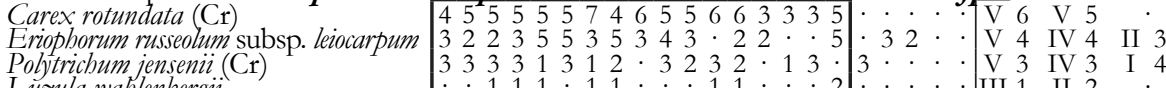

Luzula wablenbergii

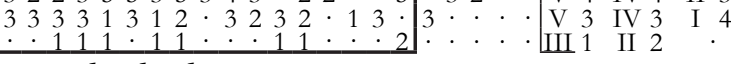

Differential species in subass. caricetosum chordorrhizae

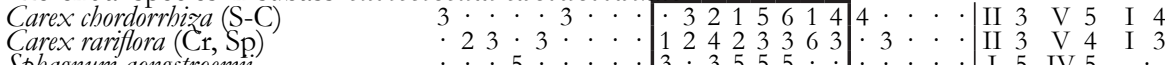
Carex rariflora (Cr, Sp)

Sphagnum aongstroemu

$23 \cdot 3$

\begin{tabular}{llllllllll}
2 & 4 & 2 & 3 & 3 & 6 & 3 \\
\hline
\end{tabular}

I 3 r

Differential species in ass. Carici stantis-Warnstorfietum exannulatae

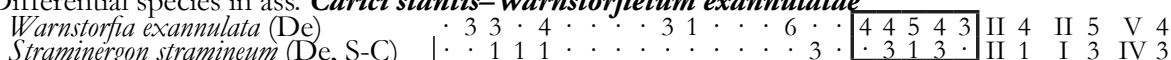

Differential species in var. Warnstorfia sarmentosa

Warnstorfia sarmentosa (De)

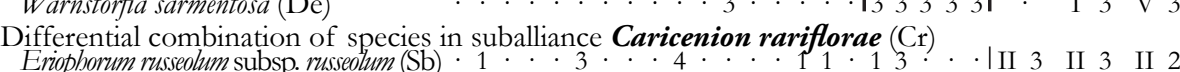

CDiagnostic species in alliance Sphagnion baltici $(\mathrm{Sb})$

Sphagnum balticum (Cr)

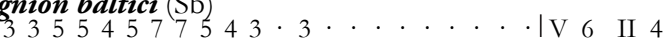
Diagnostic species in alliance Drepanocladion exannulati (De) and class Scheuchzerio-Caricetea nigrae (S-C)
Aulacomnium palustre

Comarum palustre (De)

Eriophorum angustifolium

Paludella squarrosa

Sphagnum fallax

S. lindbergii (Cr, Sb, Sp)

S. majus

S. obtusum

S. russowii

S. squarrosum

Other species

Andromeda polifolia subsp. pumila

Aulacomnium turgidum

Betula nana

Calliergon cordifolium

Dicranum laevidens

Drepanocladus aduncus

Eriophorum vaginatum

Flavocetraria cucullata

Scorpidium revolvens

Pedicularis interioroides

Salix myrtilloides

Sanionia uncinata

Sphagnum compactum

S. lenense
II 2 II 2 I 11

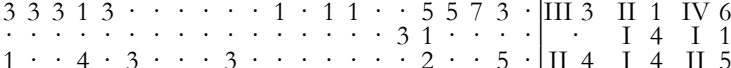

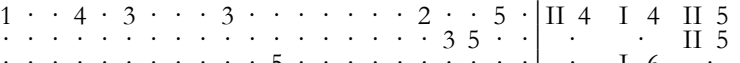

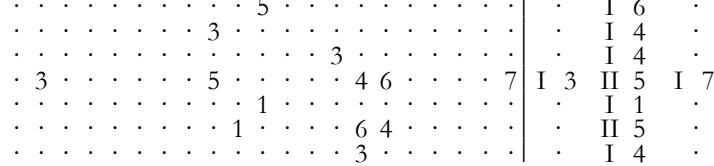

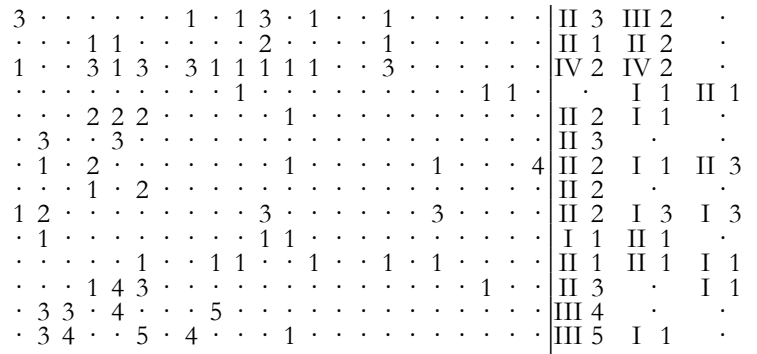

parts of their steep slopes with various aspects, at elevations between 24 and $39 \mathrm{~m}$ a.s.l. The soil organic horizon is poorly developed, peaty, $1-3 \mathrm{~cm}$ thick; the underlying mineral ground is sand.

Structure and composition. The sparse (10 to $25 \%$ ) upper layer of shrubs (Betula nana, usually $1-10 \%$ ) and herbs is usually up to $10 \mathrm{~cm}$ height. Carex arctisibirica, Hierochloë alpina, Festuca ovina, Aconogonon ochreatum, Tanacetum bipinnatum and Equisetum arvense subsp. boreale are the most abundant herbs. The dwarf shrub-cryptogam ground layer is well-developed, $1-3 \mathrm{~cm}$ thick, dominated by lichens $(20$ $80 \%$ ) and mosses (5-35\%), with patches of prostrate dwarf shrubs such as Arctous alpina, Empetrum subholarcticum, Salix nummularia (up to $35 \%$ ). Flavocetraria cucullata, Thamnolia vermicularis, Cetraria islandica, Cladonia arbuscula prevail among lichens. Polytrichum byperboreum, P. piliferum and Racomitrium lanuginosum are abundant among mosses.

\section{Low shrub communities in mesic habitats}

Dicrano maji-Salicetum lanatae ass. nova hoc loco (Table 1, rel. 15-19; Table 3; Fig. 2C)

Low willow thickets with horsetail and moss understorey we do not refer yet to any higher syntaxonomical unit. By their composition and structure, they are transitional between three classes: snowbed vegetation Salicetea herbaceae Br.-Bl. 1948, dwarf shrub tundra Loiseleurio procumbentis-Vaccinietea Eggler ex Schubert 1960 and sedge mires Scheuchzerio-Caricetea nigrae (Nordh. 1936) Tx. 1937.

Note. Single occurrence: Caltha arctica 19(1), 21(1); Dupontia fischeri 19(1), 21(1); Empetrum subholarcticum 9(1); Ledum palustre subsp. decumbens 4(1), 10(1); Pedicularis lapponica 11(1); Rubus chamaemorus 4(2), 8(2); Salix pulchra 18(1); Vaccinium uliginosum subsp. microphyllum 8(1), 9(1); V. vitisidaea subsp. minus 4(3); lichens: Cetraria islandica 6(1); C. laevioata 6(1); Cladonia cenotea 6(1); C. chlorophaea 6(1); C. cornuta 6(1); C. cyanipes 6(1); C. deformis $6(1)$; C. gracilis s.l. 6(1); C. stvgia 6(1); C. subfurcata $4(1)$; Peltigera scabrosa $4(1)$; Thamnolia vermicularis $4(1)$; mosses: Dicranum elongatum $12(4)$; D. groenlandicum 12(1); D. majus 20(3); Pohlia drummondii 18(01); Polytrichum juniperinum 11(1); Rhizomnium andrewsianum 18(1); R. pseudopunctatum 19(2); Sphagnum girgensohnii $10(4) ; S$. perfoliatum 13(5), 14(6); S. steerei $1(5), 11(3) ; S$. tundrae.

Author: Khitun O.V. Locality: Yamal-Nenets Autonomous Area. T - the Tazovsky Peninsula, upper reaches of Verkhnyaya Yareiyakha River (Fig. 1, site 1): 6 - 68 $13^{\prime} 16.9^{\prime \prime} 75^{\circ} 13^{\prime} 00.5^{\prime \prime}, 02.08 .2017 ; 7$ - 68 $13^{\prime} 15.2^{\prime \prime} 75^{\circ} 13^{\prime} 25.6^{\prime \prime}, 02.08 .2017 ; 8$ - 68 $13^{\prime} 14.0^{\prime \prime} 75^{\circ} 14^{\prime} 06.7^{\prime \prime}$, 02.08.2017; 9-

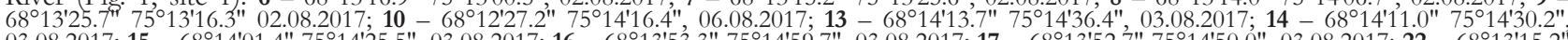

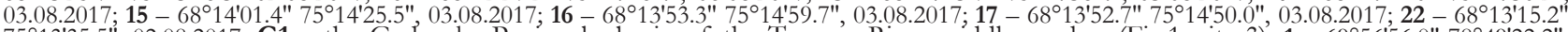
75¹3'35.5", 02.08.2017; G1 - the Gydansky Peninsula, basin of the Tanama River middle reaches (Fig.1, site 3): 1 - 69 $566^{\prime} 56.0^{\prime \prime} 78^{\circ} 49^{\prime} 22.2^{\prime \prime}$,

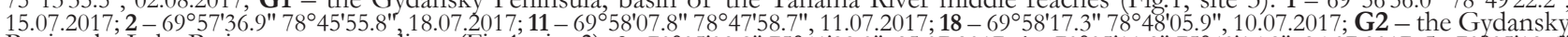
Peninsula, Lake Parisento surroundings (Fig.1, site 3): $\mathbf{3}-70^{\circ} 05^{\prime} 03.8^{\prime \prime} 75^{\circ} 41^{\prime} 22.0^{\prime \prime}, 25.07 .2017 ; 4-70^{\circ} 05^{\prime} 11.8^{\prime \prime} 75^{\circ} 40^{\prime} 16.8^{\prime \prime}, 26.07 .2017 ; \mathbf{5}^{\prime}-70^{\circ} 05^{\prime} 13.6^{\prime \prime}$

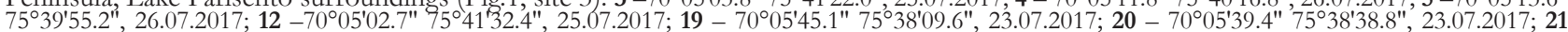
$-70^{\circ} 04^{\prime} 52.1^{\prime \prime} 75^{\circ} 43^{\prime} 03.3^{\prime \prime}, 25.07 .2017$. * - nomenclatural type. 
Differential group of species of this association are: Carex stans, Dicranum majus, Polemonium acutiflorum, Aulacomnium palustre.

Holotypus: 18 (281); T1; YaNAA; 1; 68²12'23.3"N 75²1'23.5"E; 36; 100 .

Relevé. The relevé was taken at the bottom of a hollow between two outreaches of watershed hill with a smooth surface, with an inclination of $1^{\circ}$ and an aspect of $292^{\circ}$. The cover of mosses is $45 \%$, herbs $30 \%$, shrubs $20 \%$, dwarf shrubs $10 \%$ and lichens $50 \%$. Soil organic horizon is dark brown mucky peat $15-20 \mathrm{~cm}$ thick, underlained by dark yellow sandy loam. 04.08.2017. O.V. Khitun.

List of species: Arctocetraria andrejevii 5, Cetrariella delisei 5, Polytrichum commune 4, Stereocaulon sp. 4, Betula nana 3, Cladonia gracilis s. 1. 3, Carex stans 3, Dicranum majus 3, Hylocomium splendens 3, Peltigera scabrosa 3, Salix lanata 3, S. polaris 3, Sanionia uncinata 3, Calamagrostis neglecta 2, Equisetum arvense subsp. boreale 2, Viola epipsiloides 2, Antennaria villifera 1, Aulacomnium palustre 2, Bistorta elliptica 1, B. vivipara 1, Calliergon cordifolium 1, Carex lachenalii 1, C. rariflora 1, Diphasiastrum alpinum 1, Festuca ovina 1, Ledum palustre subsp. decumbens 1, Lu₹ula multiflora subsp. frigida 1, Lycopodium annotinum subsp. pungens 1 , Pedicularic labradorica 1, Poa alpigena 1, Polemonium acutiflorum 1, Ptilidium ciliare 1, Pyrola minor 1, Salix glauca 1, Sibbaldia procumbens 1, Solidago lapponica 1, Sphagnum girgensobnii 1, Tanacetum bipinnatum 1, Trisetum spicatum 1, Vaccinium uliginosum subsp. microphyllum 1, Veratrum lobelianum 2.

Distribution and ecology. These phytocoenoses occur in the southern part of the TTS on the Gydansky Peninsula and in the STS on the Tazovsky Peninsula within an elevation range from 9 to $36 \mathrm{~m}$ a.s.l. They grow on slopes of watershed hills (inclination of 1 to $20^{\circ}$ ) and on flat bottoms of hollows with slightly hummocky nanotopography. Soil organic horizon is mucky peat underlained by sand or sandy loam.

Structure and composition. The stands have three vertical strata. The overstory is 60 to $180 \mathrm{~cm}$ high, formed by hypoarctic-montane willow species (Salix glauca, S. lanata) and Betula nana and its cover varies from 20 to $90 \%$. The field layer is 15 to $20 \mathrm{~cm}$ high, formed by herbs and varies in cover from sparse to closed $(10-70 \%)$. Herbs are distributed unevenly under the willow canopy, concentrating in open parts. Equisetum arvense dominates, Poa alpigena, Polemonium acutiflorum, Viola epipsiloides and Carex stans are common but not abundant. Dwarf shrub-moss ground layer is closed. Moss cover reaches $90 \%$; Sanionia uncinata, Hylocomium splendens and Dicranum majus dominate. Lichens and dwarf shrubs (Salix polaris and Vaccinium uliginosum subsp. microphyllum) are not abundant (5-15\%).

\section{Peat-moss vegetation in moist habitats}

Tussock tundra dominated by Eriophorum vaginatum is assigned to the class Oxycocco-Sphagnetea Br.-Bl. et Tx. ex Westhoff et al. 1946, order Sphagnetalia medii Kästner et Flössner 1933 and alliance Rubo chamaemori-Dicranion elongati O. Lavrinenko et I. Lavrinenko 2015.

The Oxycocco-Sphagnetea comprises dwarf shrub, sedge and peat-moss vegetation of the Holarctic ombrotrophic bogs, fens and wet heaths. Diagnostic species include Andromeda polifolia subsp. pumila, Aulacomnium palustre, Betula nana, Huperzia arctica, Eriophorum vaginatum, Polytrichum strictum, Rubus chamaemorus, Sphagnum balticum, S. capillifolium, S. compactum, S. fuscum, S. russowii. Vaccinium uliginosum subsp. microphyllum.

The Sphagnetalia medii is represented by Sphagnum-dominated bog communities occurring in suboceanic and continental regions, and in the mountains in the northern hemisphere. Diagnostic species are the same as for the class.
The Rubo chamaemori-Dicranion elongati includes dwarf shrub, cloudberry, moss, lichen communities on oligotrophic subarctic peatlands, including palsa bogs and high-centered polygons. Differential combination of species includes Andromeda polifolia subsp. pumila, Betula nana, Rubus chamaemorus, Ledum palustre subsp. decumbens, Vaccinium vitis-idaea subsp. minus, $V$. uliginosum subsp. microphyllum, Dicranum elongatum, Polytrichum strictum, Flavocetraria nivalis, Cetraria islandica, Cladonia arbuscula and C. rangiferina. Cladonia stygia was for a long time considered as a form of $C$. rangiferina, but now they are trated as different species (Ahti \& Hyvönen 1985) and in the studied locality we found predominantely Cladonia stygia.

We distinguished two associations.

Sphagno girgensohnii-Betuletum nanae ass. nova hoc loco (Table 1, rel. 20-22; Table 3; Fig. 2D)

Differential species: Empetrum subholarcticum, Calamagrostis neglecta, Sphagnum girgensohnii, Polytrichum commune.

Holotypus: 21 (257); T1; YaNAA; 1; 68²14'17.2"N 75¹4'48.8"E; 55; 100.

Relevé. The relevé is located on the lake's terrace with slightly hummocky surface, with an inclination $1^{\circ}$ and an aspect $225^{\circ}$. Soil organic horizon is dark brown mucky peat, $10 \mathrm{~cm}$ thick, underlain by sandy loam. The cover of mosses is $100 \%$, of shrubs $80 \%$, dwarf shrubs $10 \%$, herbs $25 \%$, lichens $20 \%$. 03.08.2017. O.V. Khitun.

List of species: Betula nana 7, Hylocomium splendens 5, Sphagnum girgensohnii 5, Cetraria islandica 4, Aulacomnium turgidum 3, Dicranum laevidens 3, Polytrichum commune 3, Rubus chamaemorus 3, Vaccinium vitis-idaea subsp. minus 3, Cladonia cornuta 2, Flavocetraria cucullata 2, Salix glauca 2, Sphenolobus minutus 1, Cetraria laevigata 1 , Cladonia cenotea 1, C. cyanipes 1 , C. pleurota 1 , C. stygia 1, Calamagrostis neglecta 1, Cladonia amaurocraea 1, Empetrum subholarcticum 1, Eriophorum vaginatum 1, Festuca ovina 1, Ledum palustre subsp. decumbens 1, Peltigera malacea 1, P. scabrosa 1 , Poa arctica 1 , Polytrichum juniperinum 1 , Ptilidium ciliare 1 , Vaccinium uliginosum subsp. microphyllum 1.

Distribution and ecology. These communities occur in the STS on the Tazovsky Peninsula at the sites with relatively deep snow cover: flat or gently sloping parts of lakes' terraces or on the edges of steep lee slopes with solifluction features in the upper parts of ravines. The soil organic horizon is $10-15 \mathrm{~cm}$ thick and consists of dark brown mucky peat; the mineral horizons are dark yellow with sandy loam texture.

Structure and composition. The canopy of Betula nana is almost closed, $70-80 \%$, and is $35-80 \mathrm{~cm}$ high. The herbal layer is $10 \mathrm{~cm}$ high, sparse, with predominance of Rubus chamaemorus and Calamagrostis neglecta. In the ground layer moss cover reaches $100 \%$, cover of lichens is 20 to $40 \%$ and of dwarf shrubs is $5-10 \%$. Sphagnum girgensobnii dominates in the moss carpet, while Hylocomium splendens is less abundant. Cetraria islandica and Cladonia cornuta are the most abundant lichens and Vaccinium vitis-idaea subsp. minus is the most abundant dwarf shrub.

Empetro subholarctici-Eriophoretum vaginati ass. nova hoc loco (Table 1, rel. 23-31; Table 3; Fig. 2E)

Differential species: Alectoria ochroleuca, Empetrum subholarcticum, Cladonia stellaris, Sphagnum lenense.

Holotypus: 27 (248); T1; YaNAA; 1; 68²13'15.5"N 75¹3'21.1"E; 47; 100.

Relevé. The stand occurs on the gentle slope of the watershed with an inclination $1^{\circ}$ and an aspect $22^{\circ}$. The surface is 
slightly hummocky and tussocky. Soil organic horizon is peaty, $17 \mathrm{~cm}$ thick, underlained by loam. The cover of lichens and mosses is $90 \%$ and $65 \%$ respectively, the cover of herbs and shrubs is 30 and $20 \%$. 02.08.2017. O.V. Khitun.

List of species: Cladonia amaurocraea 5, C. stygia 5, Sphagnum balticum 5, Ledum palustre subsp. decumbens 4, Rubus chamaemorus 4, Alectoria ochroleuca 3, Dicranum laevidens 3, Eriophorum vaginatum 3, Flavocetraria nivalis 3, Polytrichum strictum 3, Sphagnum lenense 3, Carex arctisibirica 2, Andromeda polifolia subsp. pumila 1, Calamagrostis neglecta 1, Cetraria islandica 1, C. laevigata 1, Cladonia stellaris 1, Dicranum elongatum 1, Empetrum subholarcticum 1, Vaccinium uliginosum subsp. microphyllum 1, Vaccinium vitis-idaea subsp. minus 1.

Distribution and ecology. These phytocoenoses occur on high centered polygons in the polygonal tundra-mire complexes on the flat tops of watersheds (43 to $52 \mathrm{~m}$ a.s.l.) on the Tazovsky Peninsula. Polygons are 5 to $25 \mathrm{~m}$ in diameter, 20 to $40 \mathrm{~cm}$ high and have a hummocky surface. Soil organic horison is peaty, 20 to $30 \mathrm{~cm}$ thick, underlain by sandy loam.

Structure and composition. These communities have two vertical strata. The overstory with cover of 15 to $70 \%$ is 10 to $15 \mathrm{~cm}$ high it is formed by evenly distributed graminoids and herbs, Rubus chamaemorus and Eriophorum vaginatum being most common, sometimes with low shrubs (5 to $15 \%$ ). The dwarf shrub, lichen, moss ground layer is well-developed and closed. Mosses are the most abundant between hummocks and lichens on the hummocks. Dwarf shrubs (15-30\%) are evenly distributed, Ledum palustre subsp.decum bens is the most abundant, Vaccinium uliginosum subsp. microphyllum, V. vitis-idaea subsp. minus, Empetrum subholarcticum are common but less abundant. Sphagnum balticum and Dicranum elongatum dominate among mosses, Alectoria ochroleuca, Cetraria islandica, C. laevigata, Cladonia amaurocraea, C. stygia and Flavocetraria cucullata are the most abundant among lichens.

\section{Sedge-moss vegetation in wet habitats}

Sedge-moss mires and bogs we assign to the class Scheuchzerio-Caricetea nigrae (Nordh. 1936) Tx. 1937, order Scheuchzerietalia palustris Nordh. 1936, two alliances Sphagnion baltici Kustova 1987 ex Lapshina 2010, Drepanocladion exannulati Krajina 1933 and one suballiance Caricenion rariflorae Lavrinenko, Matveyeva et Lavrinenko 2016.

The class Scheuchzerio-Caricetea nigrae comprises oligo-mesotrophic and mesotrophic bogs and intermediate mires rich in mosses and sedges. Diagnostic species of the class present in the study area are Carex chordorrbiza, C. stans, Comarum palustre, Eriophorum angustifolium, Paludella squarrosa, Sphagnum fallax, S. lindbergii, S. majus, S. obtusum, S. russowii, S. squarrosum, $S$. warnstorfii and Straminergon stramineum.
Table 3 Synoptic table of associations in the southern tundra subzone on the Tazovsky Peninsula (Syntaxa codes - see Tables 1 and 2; among "others" species with constancy $<$ II are not included).

\begin{tabular}{l|c|c|c|c|c|c|c|c}
\hline SYNTAXON CODE & A & B & C & D & E & F & G & H \\
\hline Number of relevés & 6 & 8 & 6 & 3 & 9 & 9 & 8 & 5
\end{tabular}

Diagnostic species in ass. Hierochloo alpinae-Hylocomietum splendentis Hierochloë alpina (LV) Alectoria ochroleuca (LV)

Differential species in ass. Hierochloo alpinae-Hylocomietum splendentis Festuca ovina Luzula confusa (Sh) Salix nummularia Bryocaulon divergens Flavocetraria nivalis (LV) Bryoria nitidula Racomitrium lanuginosum (Sh)

Differential species in subass. empetretosum sybholarctici and var. typicum Empetrum subbolarcticum Arctous alpina

Calamagrostis neglecta (S-C)

Differential species in var.

Tanacetum bipinnatum Aconogonon ochreatum Carex quasivaginata (S-C) Campanula rotundifotia

Differential species in ass. Dicrano maji-Salicetum lanatae Carex stans (S-C) Dicranum majus Polemonium acutiflorum Aulacomnium palustre ((O-S, S-C)

Differential species in ass. Sphagno girgensohnii-Betuletum

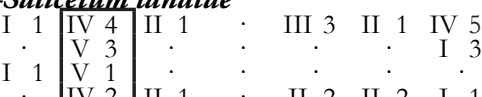
Sphagnum girgensohni Polytrichum commune II 2 -

Differential species in ass..Empetro subholarctici-Eriophoretum vaginati Cladonia stellaris (LV) Sphagnum lenense

Dif-sp. in ass. Eriophoro medii-Caricetum rotundatae and subass typicum Eriophorum medium Priophorum medium Luzula wablenbergii

Differential species in subass. caricetosum chordorrhizae Carex chordorrbiza (S-C) Carex rariflora Sphagnum aongstroemii

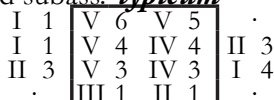

Differential species in Warnstorfia exannulata Straminergon stramineum (S-C)

Differential species in var. Warnstorfia sarmentosa Warnstorfia sarmentosa Diagnostic species in class Salicetea berbaceae (Sh)
Salix polaris Carex lachenalii Ranunculus nivalis $\begin{array}{cccc}\text { II } & 2 & \text { V } & 3 \\ \text { I } & 1 & \text { IV } & 2 \\ & \text {. } & \text { III } & 1\end{array}$

Diagnostic species in class Loiseleurio procumbentis-Vaccinietea (LV)

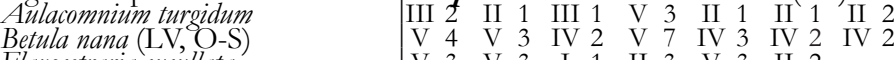

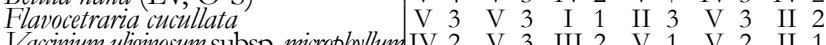

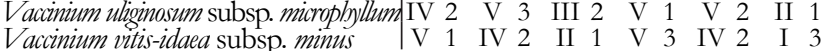
Diagnostic species in class Oxycocco-Sphagnetea (O-S)

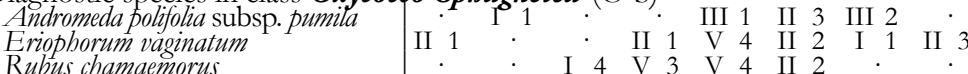
Rubus chamaemorus Sphagnum balticum S. compactum

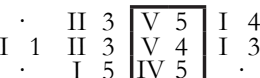
species in class Scheuchzerio-Caricetea nigrae (S-C) Eriophorum angustifolium I I III 1 II 1 I 1 II 3 I 4 II 4 Other species:

Antennaria villifera

Armeria scabra

Bistorta vivipara.

Carex arctisibirica

Cetraria islandica

C. laevigata

Cetrariella delisei

Cladonia amaurocraea

C. arbuscula

C. chlorophaea

C. coccifera

Cladonia cornuta

C. gracilis s.l.

C. pleurota

C. stygia

C. subfurcata

Dicranum elongatum

D. laevidens

Equisetum arvense sunsp. boreale

Hylocomium splendens

Ledum palustre subsp. decumbens \begin{tabular}{cccc|cc} 
II & 4 & II & 5 & V & 4 \\
II & 1 & I & 3 & III & 3 \\
\hline
\end{tabular}

I $3 \longdiv { \mathrm { V } } 3$ 
Table 3. Continued.

\begin{tabular}{|c|c|c|c|c|c|c|c|c|c|}
\hline SYNTAXON CODE & $\mathbf{A}$ & B & C & D & $\mathbf{E}$ & & $\mathbf{F}$ & G & $\mathbf{H}$ \\
\hline $\begin{array}{l}\text { Orthocaulis binsteadii } \\
\text { Peltigera scabrosa } \\
\text { Petasites frigidus } \\
\text { Pleuro:ium schreberi } \\
\text { Poa alpigena } \\
\text { Pogonatum dentatum } \\
\text { Polytrichum piliferum } \\
\text { Ptilidium ciliare } \\
\text { Pyrola minor } \\
\text { Ranunculus propinquus } \\
\text { Salix glauca } \\
\text { S. lanata } \\
\text { S. pulchra } \\
\text { Sanionia uncinata } \\
\text { Stellaria peduncularis } \\
\text { Thamnolia vermicularis } \\
\text { Veratrum lobelianum } \\
\text { Viola epipsiloides }\end{array}$ & $\begin{array}{cc}\text { III } & 1 \\
& \cdot \\
\text { I } & 1 \\
\text { II } & 1 \\
\text { III } & 1 \\
\text { I } & 1 \\
\text { I } & 1 \\
& . \\
& \cdot \\
\text { V } & 1 \\
& . \\
\text { III } & 1 \\
. \\
. \\
\text { IV } & 1\end{array}$ & $\begin{array}{cc} & \cdot \\
& \cdot \\
\text { I } & 1 \\
\text { II } & 1 \\
\text { IV } & 1 \\
\text { III } & 3 \\
\text { II } & 2 \\
& \cdot \\
\text { II } & 1 \\
\text { I } & 1 \\
\text { I } & 1 \\
\text { II } & 1 \\
\text { II } & 1 \\
\text { III } & 1\end{array}$ & $\begin{array}{cc}\text { IV } & 1 \\
\text { III } & 1 \\
\text { III } & 2 \\
\text { IV } & 3 \\
\text { V } & 5 \\
\text { I } & 1 \\
\text { V } & 5 \\
\text { III } & 1 \\
& . \\
\text { IV } & 2 \\
\text { III } & 2\end{array}$ & $\begin{array}{c}\cdot \\
\mathrm{V} 1 \\
: \\
\text { IV } 2 \\
\text { II } 1 \\
\cdot \\
\text { II } 3 \\
: \\
\cdot \\
\cdot \\
\cdot\end{array}$ & $\begin{array}{cc}\text { II } & 1 \\
& \cdot \\
\text { II } & 2 \\
& \cdot \\
& \cdot \\
& \cdot \\
\text { II } & 1 \\
& \cdot \\
& \cdot \\
& \cdot \\
\text { I } & 1 \\
\text { I } & 1 \\
\text { II } & 1 \\
& \cdot \\
\text { I } & 3\end{array}$ & I & 1 & $\begin{array}{l}. \\
. \\
. \\
. \\
. \\
. \\
.\end{array}$ & 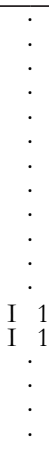 \\
\hline
\end{tabular}

soils are waterlogged, with peat horizons 20 to $30 \mathrm{~cm}$ thick underlained by sandy loam or clay loam.

Structure and composition. The stands are rather homogenous, formed by hygrophitic graminoids and predominantly Sphagnum mosses. The layer of graminoids in these communities is up to $80 \mathrm{~cm}$ high, with the cover of 40 to $85 \%$. Carex rotundata, Eriophorum russeolum subsp. leiocarpum and Luqula wablenbergii are the most abundant. Hygrophytic mosses (Sphagnum balticum and Polytrichum jensenii predominate) form a thick carpet with 40 to $100 \%$ cover. Rarely small patches of dwarf shrubs are present on the moss pads (Andromeda polifolia subsp. pumila, Ledum palustre subsp. decumbens).
The Scheuchzerietalia palustris comprises wet ombrotrophic and minerotrophic communities in depressions in bogs, swamps and mires occurring on peaty soil with high water table. Diagnostic species: Carex rariflora and Sphagnum lindbergii.

The Sphagnion baltici comprises vegetation of wet depressions in bogs and intermediate mires dominated by oligotrophic Sphagnum-mosses in continental parts of the boreal zone in the Holarctic. Diagnostic species are: Sphagnum balticum, S. lindbergii and Eriophorum russeolum (Lavrinenko et al. 2016). The alliance is represented by suballiance Caricenion rariflorae, comprising vegetation of swamps and depressions in polygonal bogs and palsas dominated by oligotrophic Sphagnum-mosses in the tundra zone of the East European and probably West Siberian sectors of the Arctic. Differential combination of species includes Carex rariflora, C. rotundata, Eriophorum russeolum, Sphagnum balticum, S. lindbergii and Polytrichum jensenii (Lavrinenko et al. 2016).

We describe one new association and two subassociations in this suballiance.

Eriophoro leiocarpi-Caricetum rotundatae (Table 2, rel. 1-17; Table 3)

Differential species: Carex rotundata, Eriophorum russeolum subsp. leiocarpum, Polytrichum jensenii, Luzula wablenbergii.

Holotypus: 4 (204); T2; YaNAA; 2; 7005'11.8" 7540'16.8" E; 20; 100.

Relevé. The community occurs in the water track channel on the gentle slope. The cover of mosses is $90 \%$. The cover of graminoids is $80 \%$, shrubs and dwarf shrubs $5 \%$ each. Soils are peaty, moist, with $15-20 \mathrm{~cm}$ thick organic horizon underlained by sandy loam. 26.07.2017. O.V. Khitun.

List of species: Carex rotundata 5, Sphagnum aongstroemii 5, S. balticum 5, Eriophorum angustifolium 4, Betula nana 3, Dicranum laevidens 2, Eriophorum russeolum subsp. leiocarpum 3, E. vaginatum 3, Polytrichum jensenii 3, Vaccinium vitis-idaea subsp. minus 3, Aulacomnium turgidum 1, Carex stans 1, Cladonia subfurcata 1, Flavocetraria cucullata 1, Ledum palustre subsp. decumbens 1, Luzula wablenbergii 1, Peltigera scabrosa 1, Rubus chamaemorus 1, Sanionia uncinata 1, Straminergon stramineum 1, Thamnolia vermicularis 1.

Distribution and ecology. These communities occur in the southern part of the TTS on the Gydansky Peninsula and in the STS on the Tazovsky Peninsula. They occupy lake depressions, drained lakebeds, wet hollows between watershed hills, wet troughs on the watershed hills. The
Eriophoro leiocarpi-Caricetum rotundatae typicum subass. nov. hoc loco (Table 2, rel. 1-9; Table 3; Fig. 2F)

Differential species are the same as for the association.

Distribution and ecology. These phytocoenoses occur in the southern part of the TTS on the Gydansky Peninsula and in the STS on the Tazovsky Peninsula within the altitude range of 17 to $52 \mathrm{~m}$ a.s.l. on the drained lakebeds, on low poorly drained watersheds, in troughs of polygonal tundra-mire complexes.

Eriophoro leiocarpi-Caricetum rotundatae caricetosum chordorrbizae subass. nov. hoc loco (Tabl. 2, rel. 10-17; Tabl. 3; Fig. 2G)

Differential species: Carex chordorrbiza, C. rotundata, C. rariflora and Sphagnum aongstroemii.

Holotypus: 13 (261); T2; YaNAA; 1; 68¹4'13.7"N 75²14'36.4"E; 51; 100.

Relevé. The stand grows on drained lakebed with a rather even surface. The cover of mosses is $100 \%$, graminoids $80 \%$, shrubs and dwarf shrubs $1 \%$ each. Soils are peaty and wet, with organic horizon $20-25 \mathrm{~cm}$ thick, underlained by sandy loam. 03.08.2017. O.V. Khitun.

List of species: Carex rotundata 6, Sphagnum aongstroemii 5, Sphagnum perfoliatum 5, Sphagnum balticum 3, Carex rariflora 2, Eriophorum russeolum subsp. leiocarpum 2, Polytrichum jensenii 2, Andromeda polifolia subsp. pumila 1, Betula nana 1, Carex chordorrbiza 1, Luzula wablenbergii 1, Salix myrtilloides 1.

Distribution and ecology. These communities occur in the southern part of the T'TS on the Gydansky Peninsula and in the STS on the Tazovsky Peninsula. They occupy drained lakebeds, seldom occur in troughs of polygonal complexes on the lower watersheds. They were recorded within the altitude range of 17 to $54 \mathrm{~m}$ a.s.l.

Structure and composition. Two vertical strata are clearly distinguished in this community: an upper layer of hygrophitic sedges (Carex rotundata, C. chordorrbiza, C rariflira) 20 to $30 \mathrm{~cm}$ high with cover 50 to $100 \%$, and a closed ground layer (100\%) formed by hygrophitic mosses Polytrichum jensenii and Sphagnum aongstroemii.

The alliance Drepanocladion exannulati comprises non-calcyphytic fens dominated by mosses (Warnstorfia exannulata and $W$. sarmentosa) and with a sparse sedge cover (Eriophorum polystachion, E. scheuchzeri, Carex cinerea, C. lachenalii, C. rariflora, C. rostrata). Communities of this alliance occur in boreal and arctic regions as well as in alpine areas in Central and Southern Europe. Diagnostic species include Comarum pa- 
lustre, Straminergon stramineum, Warnstorfia exannulata, W. sarmentosa. In the study area this alliance is represented by the variant of the association Carici stantis-Warnstorfietum exannulatae Lavrinenko, Matveyeva et Lavrinenko 2016, described earlier in the East European Arctic (Lavrinenko et al. 2016).

Carici stantis-Warnstorfietum exannulatae Lavrinenko, Matveyeva et Lavrinenko 2016

Differential combination of species includes Carex stans, Warnstorfia exannulata and Straminergon stramineum.

Total vegetation cover is $80-100 \%$. Sedges and solitary willows form the canopy up to $30 \mathrm{~cm}$ high, with the cover from 35 to $95 \%$. The ground moss layer varies from $1 \%$ to almost closed. These stands occupy paludified depressions on watersheds, low marine terraces and floodplains. The soils are peaty, underlained by gleyed mineral horizons. Association is present in the TTS and STS of the East European Arctic (Lavrinenko et al. 2016). We describe a variant of this association below.

Carici stantis-Warnstorfietum exannulatae var. Warnstorfia sarmentosa (Tabl. 2, rel 18-22; Tabl. 3; Fig. 2H).

Differential species: Warnstorfia sarmentosa.

Distribution and ecology. These communities occur in the southern part of the TTS on the Gydansky Peninsula and in the STS on the Tazovsky Peninsula. They were recorded within an altitude range of 21 to $44 \mathrm{~m}$ a.s.l. in waterlogged habitats at the bottoms of hollows between hills, on drained lakebeds or in thermocarst thaw troughs on watersheds. The soils with approximately $15 \mathrm{~cm}$ thick peat horizon, water-soaked, on sandy deposits.

Structure and composition. The stands have two vertical layers: Carex stans dominates in the upper one, which height is $30-35 \mathrm{~cm}$ and cover up to $80 \%$; Warnstorfia exannulata, $W$. sarmentosa and Straminergon stramineum are predominant in the ground layer which cover varies from 40 to $100 \%$.

\section{Overview of the syntaxonomical diversity of the Tazovsky Peninsula and the prodromus of vegetation}

For the first time, for the STS on the Tazovsky Peninsula, the syntaxonomic diversity is described, including 6 associations, 3 subassociations and 3 variants. Among them 4 associations, 3 subassociations and 3 variants are nov. hoc. loco. The subass. Hierocbloo alpinae-Hylocomietum splendentis empetretosum subholarctici with 2 variants the typicum and the Aconogonon ochreatum are wide-spread and occupy the most drained parts of the watershed hills and their edges. The association Dicrano maji-Salicetum lanatae is common in the study area but occupy relatively small areas on the slopes of watersheds and hollows between them. The association Sphagno girgensohnii-Betuletum nanae was relatively rarily found, mainly on the gently sloping lake terraces. The tussock tundra Empetro subholarctici-Eriophoretum vaginati and the sedge-Sphagnum mires Eriophoro leiocarpi-Caricetum rotundatae are the parts of polygonal tundra-mire complexes on the flat watersheds. The sedge-moss fens (subass. Carici stantis-Warnstorfietum exannulatae warnstorfietosum sarmentosae) in lake depressions and hollows betwen hills are rather common in the area.

A hyerarchy of the syntaxonomical system of vegetation of the Tazovsky Peninsula is represented in the following prodromus:
Class

Order

Alliance

Suballiance

Association

Subassociation

Variant

Loiseleurio procumbentis-Vaccinietea Eggler ex Schubert 1960

Deschampsio flexuosae-Vaccinietalia myrtilli Dahl 1957

Loiseleurio-Arctostaphylion Kalliola ex

Nordhagen1943

Hierochloo alpinae-Hylocomietum splendentis

Telyatnikov et al. 2019

empetretosum subbolarctici Khitun subass. nov. hoc loco

typicum Khitun var. nov. hoc loco

Aconogonon ochreatum Khitun var. nov. hoc loco

? Unknown class

? Unknown order

? Unknown alliance

Dicrano maji-Salicetum lanatae Khitun ass. nov. hoc loco

Oxycocco-Sphagnetea Br.-Bl. et Tx. ex Westhoff et al. 1946

Sphagnetalia medii Kästner et Flössner 1933

Rubo chamaemori-Dicranion elongati

O. Lavrinenko et I. Lavrinenko 2015

Sphagno girgensobnii-Betuletum nanae Khitun ass. nov. hoc loco

Empetro subbolarctici-Eriophoretum vaginati Khitun ass. nov. hoc loco

Scheuchzerio-Caricetea nigrae (Nordh. 1936) Tx. 1937

Scheuchzerietalia palustris Nordh. 1936

Sphagnion baltici Kustova 1987 ex Lapshina 2010

Caricenion rariflorae Lavrinenko, Matveyeva et

Lavrinenko 2016

Eriophoro leiocarpi-Caricetum rotundatae

Khitun ass. nov. hoc loco

typicum Khitun subass. nov. hoc loco caricetosum chordorrhizae Khitun subass. nov. hoc loco

Drepanocladion exannulati Krajina 1933

Carici stantis-Warnstorfietum exannulatae

Lavrinenko, Matveyeva et Lavrinenko 2016

Warnstorfia sarmentosa Khitun var. nov. hoc loco

\section{DISCUSSION}

In the STS on the Tazovsky Peninsula we found the same tendencies as in the southern part of the TTS on the Gydansky Peninsula. (Telyatnikov et al., in press). As expected, spotted dwarf shrub-moss tundras with notable participation of forbs (subass. Luzulo tundricolae-Hylocomietum splendentis empetretosum subbolarctici), which are characteristic for the zonal habitats in the northern part of the TTS on the Gydansky Peninsula, are absent on Tazovsky. On the Tazovsky Peninsula, dwarf birch dominated communities with an abundance of erect dwarf shrubs, sedge (Carex arctisibirica) and well-developed lichen-moss ground layer with rare frost boil spots occupy zonal habitats on loamy grounds (Khitun 1989). However, in the studied locality sandy grounds were absolutely predominant, therefore we collected not enought relevés corresponding strictly to this zonal type to describe it here. Instead, psammophitic variants of such communities were present. We assigned them to the earlier described association Hierocbloo alpinae-Hyloco- 
mietum splendentis. These phytocoenoses grow on the better drained parts of watershed hills with shallow peaty horizon.

On the poorly drained parts of watershed hills on sandy loams or loams, where peaty horizon is rather thick, tussock tundras occur (ass. Empetro subholarctici-Eriophoretum vaginati). In the southern part of the TTS in the central part of the Gydansky Peninsula tussock tundra is widespread and we considered it as a zonal community (Telyatnikov et al., in press). This idea goes back to Gorodkov (1935). Matveyeva \& Zanokha (1986) in their surveys of vegetation in the southern tundra of the Taimyr Peninsula also referred communities with abundant Eriophorum vaginatum to the zonal type.

Though tussock tundra is physiognomically very similar and have similar species composition in various regions, some regional or local variation is present (Walker et al. 1994). For example, Cassiope tetragona, common in this type of tundra in Taimyr, was not found in similar habitats in the West Siberian Arctic. We found some difference in dominant species of Sphagnum mosses. In the central part of the Gydansky Peninsula, Sphagnum balticum and $S$. aongstroemii were dominant in tussock tundra, the latter is also the main dominant in tussock tundra in the STS of Taimyr (Matveeva \& Zanokha 1986). On the Tazovsky Peninsula, Sphagnum balticum and $S$. lenense dominate in the tussock tundra. In the study area we found Sphagnum aongstroemii only on the former lakebeds of drained lakes in the wet sedge-moss mires.

Tussock tundra usually develops on loam and clay grounds and is characterized by a poorer drainage. However, in the studied localities sand deposits were widespread, so sandy loams and sands were underlaying relatively thick organic peaty horizons. It is likely that these peat horizons provide the conditions suitable for such communities. The presence of thick peaty horizons is very characteristic of subarctic tundra in the West Siberian Arctic (Rebristaya 2013, Khitun 2005). Peaty acidic soils are favourable for many oligotrophic hypoarctic species, but are an obstacle to the spread of arctic species southwards (in contrast to Taimyr, where arctic species are present even in the southern tundra). The absence of arctic species is the main reason that the species richness of zonal communities in the West Siberian Arctic is lower, when compared to Taimyr; and the tussock tundras are the poorest of the zonal communities in the region (by species composition). Only in the subzone of the arctic tundra, where organic horizons are minimal and mineral horizons are close to the surface, we recorded the increase of forb species and zonal communities become one of the richest by number of species (Khitun 1998, 2005).

In the studied locality, as well as in the localities in the southern part of the TTS on the Gydansky Peninsula, we did not find Dryas-dominated communities Dryado octopetalae-Hylocomietum alaskani (Andreev 1932) Lavrinenko et Lavrinenko 2018, which was unexpected, taking into account the widespread sandy grounds. It is probable that the thick peat horizons and poor drainage, overall, was the reason for their absence. In previously studied sites on the Tazovsky Peninsula Dryas-dominated stands were also rare (Khitun 1989).

Vegetation in the southern part of the TTS on the Gydansky and in the STS on the Tazovsky Peninsulas is phyto- coenologically linked with the territories located westwards. Association Carici stantis-Warnstorfietum exannulatae is common with the East-European Arctic but there are no common associations with regions located to the east. In the northern part of the TTS on the Gydansky Peninsula, we discovered the opposite trend, finding connections only with regions located eastward, i.e. Taimyr, East Siberia (Telyatnikov et al. 2019).

We found a high degree of similarity between vegetation of Tazovsky and southern part of the TTS of the Gydansky Peninsula. Four of six associations which we described are common for these territories (Hierochloo alpinae-Hylocomietum splendentis, Dicrano maji-Salicetum lanatae, Eriophoro leiocarpi-Caricetum rotundatae, Carici stantis-Warnstorfietum exannulatae) and only two (Sphagno girgensohnii-Betuletum nanae and Empetro subholarctici-Eriophoretum vaginati) are specific for the STS on the Tazovsky Peninsula. At the same time, comparison of the northern part of the TTS and STS, revealed low similarity with only one association in common (Hierochloo alpinae-Hylocomietum splendentis).

\section{CONCLUSION}

Summarizing the results of our phytosociological research in the Gydansky and Tazovsky Peninsulas, we conclude that the results correspond with the statements of Alexandrova (1980), who wrote that the vegetation of the northern part of the TTS differed notably from the vegetation of the southern parts of the TTS and of STS. The two latter are rather similar by species composition and structure. According to Alexandrova (1980), zonal communities in the northern belt of the subarctic tundra (coinciding with northern part of TTS) have the features transitional to the arctic tundra subzone, but the presence of low shrubs (dwarf birch and willows) is characteristic for subarctic tundra. Our data confirm this conclusion.

\section{ACKNOWLEDGEMENTS}

The authors are grateful to Transregional Expeditional Center "Arctica", Scientific Center of Arctic Studies and Department of Science and Innovations of the YamalNenets Autonomous Area for the field work logistics. We are very thankful to Dr. Il'ya Zhdanov for identification of lichens in the field, to our cartographer Yakov Gunin for various help during the field work and to Bernard Steward for improving the English language text. The work was carried out with funding of the State Task of the Central Siberian Botanical Garden Siberian Branch RAS (№ AAAA-A21-121011100007-6), and with the support of the RFBR (project no 18-04- 01010 A). Work of the reseachers of the Komarov Botanical Institute RAS was carried out within the framework of the institute topics № AAAA-A19-119030690002-5 and № 121021600184-6, and support of RFBR project no 18-05-60093.

\section{LITERATURE CITED}

Ahti, T. \& S. Hyvönen 1985. Cladyna stygia, a common, overlooked species of reindeer lichen. Annals Botanici Fennici 22:223-229.

Aleksandrova, V.D. 1980. The Arctic and Antarctic: their division into geobotanical areas. Cambrige Univ. Press, Cambrige, 247 pp. 
Antonov, V.M. et al. (eds) 1986. Atlas of the USSR. Moscow, 260 pp. (in Russian). [Атлас СССР / пол реА. В.М. Антонова и Ар. 1986. М., 260 с.].

Bogolyubov, A.S., M.V. Kravchenko \& S.V. Baslerov 2001. The simplest method of describing the soil. Moscow, 16 pp. (in Russian). ББоголюбов А.С., Кравченко М.В., Баслеров С.В. 2001. Простейшая методика описания почв. М., 16 с.].

CAVM Team. 2003. Circumpolar Arctic Vegetation Map. Conservation of Arctic Flora and Fauna (CAFF) Map No. 1. Anchorage, AK, US: U.S. Fish and Wildlife Service.

Chernov, Yu.I. \& Matveyeva N.V. 1997. Arctic ecosystems in Russia. In: Ecosystems of the World 3, Polar and Alpine Tundra (F.E. Wielgolaski, ed.), pp. 361-507, Elseiver, Amsterdam.

Ermakov, N.B. 2012. Prodromus of higher vegetation units of Russia. In: Modern state of the basic concepts of Vegetation Science (B.M. Mirkin \& L.G. Naumova), pp. 377-483, Gilem, Ufa (in Russian). [Ермаков Н.Б. 2012. Продромус высших единиц растительности России // Миркин Б.М., Наумова А.Г. Современное состояние основных концепций науки о растительности. Уфа: Гилем. С. 377-483].

Esslinger, T.L. 2016. A cumulative checklist for the lichenforming, lichenicolous and allied fungi of the Continental United States and Canada, Version 21. Opuscula Philolichenum 15:136-390.

Gorodkov, B.N. 1935. Vegetation of the USSR tundra zone. Moscow, Leningrad, 142 pp. (in Russian). [Гордков Б.Н. 1935. Растительность тунаровой зоны СССР. Москва; МенинграА, 142 с.].

Govorukhin, V.S. 1933. Essay on the vegetation of summer reindeer pastures in the tundra of the Ob-Taz Peninsula. Zemlevedenie 35(1):68-92 (in Russian). [Говорухин B.C. 1933. Очерк растительности метних пастбищ северного оленя в тунарах Обско-Тазовского полуострова // Землеведение. Т. 35, вып. 1. С.68-92].

Hennekens, S.M. \& J.H.J. Schaminée 2001. TURBOVEG, a comprehensive database management system for vegetation data. Journal of Vegetation Science 12:589-591.

Hennekens, S.M. 1996. MEGATAB a visual editor for phytosociological tables. Uift, $11 \mathrm{pp}$.

Hill, M.O. 1979. DECORANA and TWINSPAN, for ordination and classification of multivariate species data: a new edition, together with supporting programs, in FORTR AN 77. Huntingdon, $58 \mathrm{pp}$.

Ignatov, M.S., O.M. Afonina, E.A. Ignatova, A.A. Abolinya, T.V. Akatova, E.Z. Baisheva, L.V. Bardunov et al. 2006. Check-list of mosses of East Europe and North Asia. Arctoa 15:1-130 (in Russian with English summary). [Игнатов М.С., Афонина О.М., Игнатова Е.А., Аболиня А.А., Акатова Т.В., Баишева Э.З., Бардунов А.В. и Ар. 2006. Список мхов Восточной Европы и Северной Азии // Arctoa. T. 15. С. 1-130].

Il'ina, I.S. (ed.) 1976. Vegetation of the West Siberian Plain. A map. M 1: 500 000. GUGK, Moscow (in Russian). [Pacтительность Западно-Сибирской равнины. Карта М 1: 500 000. 1976 / поА реА. И.С. ИАьиной. М.: ГУГК.]

Il'ina, I.S., E.I. Lapshina, N.N. Lavrenko, L.I. Melzer, E.A. Romanova, B.A. Bogoyavlensky \& V.D. Makhno 1985. The vegetation cover of the West Siberian Plain. Nauka, Novosibirsk, 251 pp. (in Russian). [ИАьина И.С., Аапшина Е.И., Аавренко Н.Н., Мемьцер А.И., Романова Е.А., Богоявленский Б.А., Махно В.А. 1985. Растительный покров ЗапаАно-Сибирской равнины. Новосибирск: Наука. 251 с.].

Khitun, O.V. 1989. Floristic characteristics of habitas in two local floras of the Tazovsky peninsula (the Western
Siberia). Botanicheskii Zhurnal 74(10):1466-1476 (in Russian with English summary). [Хитун О.В. 1989. ФАористическая характеристика экотопов Авух мокальных фцор на Тазовском полуострове (Западная Сибирь) // Ботанический журнал. Т. 74, № 10. С. 1466-1476].

Khitun, O.V. 1998. Comparative analysis of local and partial floras in two subzones of the West Siberian Arctic (Gydansky and Tazovsky peninsulas). In: The study of biological diversity by methods of comparative floristics (B.A.Yurtsev, ed.), pp. 173-201, St. Petersburg [in Russian]. (Хитун O.B. Сравнительный анализ мокальных и парциальных флор в Авух подзонах Западносибирской Арктики (п-ова Гыданский и Тазовский) // Изучение биологического разнообразия методами сравнительной фмористики / под реА. Б.А. Юрцева. СПб., 1998. С. 173-201).

Khitun, O.V. 2005. Zonal and ecotopological differentiation of the flora of the central part of the West Siberian Arctic (Gydansky and Tazovsky Peninsulas). Abstract dis. cand. biol. sciences. Saint Petersburg, 28 pp. (in Russian). [Хитун O.B. 2005. Зональная и экотопологическая Аифференциация флоры центральной части Западносибирской Арктики (Гыданский и Тазовский полуострова): Автореферат Аиссертации кандидата биологических наук. Санкт Петерборг. 28 с.].

Lavrinenko, O.V \& I.A. Lavrinenko 2015. Communities of the class Oxycocco-Sphagnetea Br.-Bl. et R. Tx. 1943 in the East European tundras. Rastitel'nost' Rossii 26:55-84 (in Russian with English summary). [ $А$ авриненко O.B., Аавриненко И.А. 2015. Сообщества класса ОхусоссоSphagnetea Br.-Bl. et R. Tx. 1943 в в восточноевропейских тунарах // Растительность России. № 26. С. 55-84].

Lavrinenko, O.V., N.V. Matveyeva \& I.A. Lavrinenko 2016. Communities of the class Schenchzerio-Caricetea nigrae (Nordh. 1936) Tx. 1937 in the East European tundras. Rastitel'nost' Rossii 28:55-88 (in Russian with English summary). [Аавриненко О.В., Матвеева Н.В., Аавриненко И.А. 2016. Сообщества класса Scheuchzerio-Caricetea nigrae (Nordh. 1936) Тх. 1937 в восточноевропейских тундрах // Растительность России. № 28. С. 55-88].

Matveyeva, N.V. \& L.L. Zanokha 1986. Vegetation of the southern tundra in the western Taimyr. In: Southern tundras of Taimyr (Yu.I. Chernov \& N.V. Matveeva, eds), pp. 5-67, Nauka, Leningrad (in Russian). [Матвеева Н.B, Заноха

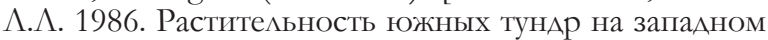
Таймыре / / Южные тундры Таймыра / отв. реА. Ю.И. Чернов и Н.В. Матвеева. АенинграА: Наука. С. 5-67].

Mirkin, B.M. \& L.G. Naumova 1998. Vegetation science (bistory and current state of the basic concepts), Gilem, Ufa, $410 \mathrm{pp}$. (in Russian). [Миркин Б.М., Наумова А.Г. 1998. Наука о растительности (история и современное состояние основных концепций). Уфа: Гилем. 410 с.].

Moskovchenko, D.V., S.P. Arefyev, V.A. Glazunov \& A.A. Tigeev 2017. Changes in vegetation and geocryological conditions of the Tazovsky peninsula (eastern part) for the period of 1988-2016. Kriosfera Zemli 21(6):3-13 (in Russian with English summary) [Московченко А.B., Арефьев С.П., ГАазунов В.А., Тигеев А.А. 2017. Изменение состояния растительности и геокриологических условий Тазовского полуострова (восточная часть) за период 1988-2016 гг. // Криосфера Земли, T. 21, № 6. C. 3-13].

Moskovchenko, D.V., V.A. Glazunov \& A.A. Tigeev 2016. Study of vegetation cover changes on the eastern part of the Tazovsky peninsula. Ekologicheskii Monitoring i Bioraznoobrazie (11)1:91-96 (in Russian). [Московченко А.B., ГАазунов В.А., Тигеев А.А. 2016. Исследование Аинамики растительного покрова восточной части Тазов- 
ского полуострова // Экологический мониторинг и биоразнообразие. Т. 11, № 1. С. 91-96].

Mucina, L., H. Bültmann, K. Dierßen, J.-P. Theurillat, T. Raus, A. Čarni, K. Šumberová, et al. 2016. Vegetation of Europe: hierarchical floristic classification system of vascular plant, bryophyte, lichen, and algal communities. Applied Vegetation Science 19(1): 3-264.

Nikolaeva, M.G. 1941. Shrub vegetation type of the Bolshoi and Malyi Yamal. Botanicheskii Zhurnal 26(1):52-86 (in Russian with German resume). Николаева М.Г. 1941. Кустарниковый тип растительности Большого и Малого Ямала // Ботанический журнал. Т. 26, № 1. С. 52-86).

Ogorodnov, E.A. et al. (eds) 1971. Atlas of the Tyumen Region. Tyumen. Issue 1.198 pp. (in Russian). [Атлас Тюменской области. 1971 / отв. реА. Е.А. Огороднов и Ар. Тюмень. Вып. 1. 198 с.].

Potemkin, A.D. \& E.V. Sofronova 2009 Liverworts and hornworts of Russia, vol. 1. Saint Petersburg, Yakutsk. 368 pp. [Потёмкин А. А., Софронова Е. В. 2009. Печеночники и антоцеротовые России. СПб; Якутск. Т. 1. 368 с.].

Rebristaya O.V. 2013. Flora of the Yamal peninsula. Modern state and history of the formation. LETI, Saint Petersburg, $312 \mathrm{pp}$. (in Russian with English summary). РРебристая O.B. 2013. ФАора полуострова Ямац. Современное состояние и история формирования. Санкт-Петербург: АЭТИ, 312 с.].

Rebristaya, O.V., V.A. Tvorogov \& O.V. Khitun 1989. Flora of the Tazovsky peninsula (the north of the Western Siberia). Botanicheskii Zhurnal 74(1):22-35. (in Russian with English summary). [Ребристая О.В., Творогов В.А., Хитун О.В. 1989. ФАора Тазовского полуострова (север Западной Сибири) // Ботанический журнал. T. 74, № 1. C. 22-35].

Sekretareva, N.A. 2004. Vascular plants of Russian Arctic and adjacent territories. KMK Press, Moscow, 131 pp. (in Russian). [Секретарева Н.А. 2004. Сосудистые растения Российской Арктики и сопредельных территорий. М.: Иза-во КМК 131 с.].

Simonov, I.M. 1977. Climate. In: The Yamal-Gydan Region (R.K. Sisko, ed.), pp. 27-50, Gidrometeoizdat, Leningrad (in Russian). [Симонов И.М. 1977. КАимат // ЯмацоГыланская область / под реА. Р.К. Сиско. АенинграА: Гидрометеоиздат. С. 27-50].

Telyatnikov, M.Yu., E.I. Troeva, K.A. Ermokhina \& S.A. Pristyazhnyuk 2019. Vegetation of the two regions of the northern part of the Gydan Peninsula (the subzone of typical tundras). Turczaninowia 22(4):128-144 (in Russian with English summary). (Телятников М.Ю., Троева Е.И., Ермохина К.А., Пристяжнюк С.А. 2019. Растительность Авух районов северной части ГыАанского полуострова (подзона типичных тундр) // Turczaninowia. Т. 22, вып. 4. С. 128-144).

Theurillat, J.-P., W. Willner, F. Fernández-González, H. Bültmann, A. Carni, D. Gigante, L. Mucina \& H. Weber. 2021. International Code of Phytosociological Nomenclature. 4th edition. Applied Vegetation Science 24: e12491.

Valeyeva, E.I. \& D.V. Moskovchenko 2008. Zonal features of the vegetation cover of the Taz Peninsula and its technogenic transformation. Vestnik Ekologii, Lesovedeniya $i$ Landshaftovedeniya 9:174-191 (in Russian with English summary). (Валеева Э.И., Московченко А.В. 2008. Зональные особенности растительного покрова Тазовского полуострова и его техногенная трансформация // Вестник экологии, Аесоведения и манАшафтоведения. Т. 9. С. 174-191).

Walker, M.D., D.A. Walker \& N.A. Auerbach 1994. Plant communities of a tussock tundra landscape in the Brooks Range Foothills, Alaska. Journal of Vegetation Science 5-6: 843-866.

Walker, D.A., FJ.A. Daniëls, N.V. Matveyeva, J. Šibík, M.D. Walker, A.L. Breen, L.A. Druckenmiller, M. Raynolds al. 2018. Circumpolar Arctic Vegetation Classification. Phytocoenologia 48(2):181-201.

Westhoff, V. \& E. van der Maarel 1973. The Braun-Blanquet approach. In: Handbook of vegetation science. Part 5 (R. Tüxen, ed.), pp. 617-726, Junk, Amsterdam.

Yurtsev, B.A. 1994. Floristic division of the Arctic. Journal of Vegetation Science 5-6:765-774. 